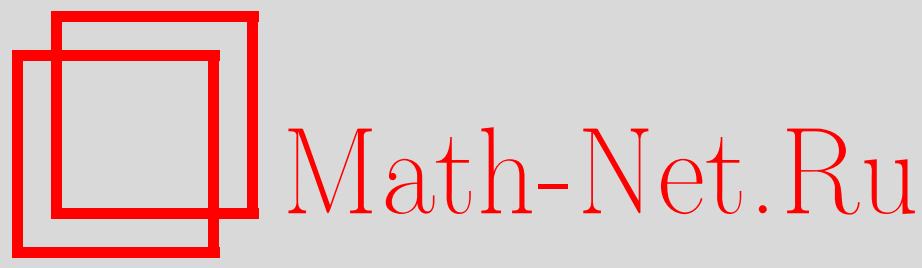

В. В. Пупышев, Двумерное ядерно-кулоновское рассеяние медленной квантовой частицы, ТМФ, 2017, том 193, номер 2, 225-255

DOI: https://doi.org/10.4213/tmf9270

Использование Общероссийского математического портала Math-Net.Ru подразумевает, что вы прочитали и согласны с пользовательским соглашением http://www . mathnet.ru/rus/agreement

Параметры загрузки:

IP: 3.91 .87 .62

26 апреля 2023 г., 15:09:12

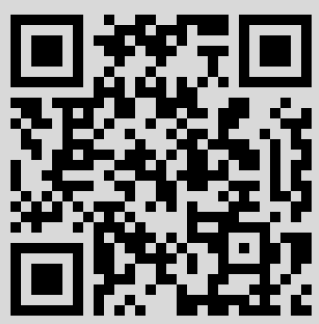




\title{
ДВУМЕРНОЕ ЯДЕРНО-КУЛОНОВСКОЕ РАССЕЯНИЕ МЕДЛЕННОЙ КВАНТОВОЙ ЧАСТИЦЫ
}

\begin{abstract}
Исследуется двумерное рассеяние квантовой частицы суперпозицией кулоновского и центрального короткодействующего потенциалов. Дан анализ низкоэнергетических асимптотик всех радиальных волновых функций, парциальных фаз и сечений рассеяния такой частицы. Предложены два способа вычисления длины рассеяния и эффективного радиуса.
\end{abstract}

Ключевые слова: двумерное рассеяние, суперпозиция центрального короткодействующего и кулоновского потенциалов, низкоэнергетические асимптотики, длина рассеяния, эффективный радиус.

DOI: https://doi.org/10.4213/tmf9270

\section{1. ВВЕДЕНИЕ}

Объект нашего исследования - упругое рассеяние квантовой частицы $p_{1}$ в двумерной плоскости $\mathcal{P}$ неподвижным силовым центром $O$, лежащим в той же плоскости и воздействующим на эту частицу посредством суперпозиции $V^{\mathrm{c}}+V^{\mathrm{s}}$ кулоновского потенциала $V^{\mathrm{c}}$ и центрального короткодействующего потенциала $V^{\mathrm{s}}$. Следуя терминологии, принятой в ядерной физике, такое рассеяние назовем двумерным ядерно-кулоновским рассеянием.

Двумерное ядерно-кулоновское рассеяние и его низкоэнергетические параметры, длина рассеяния и эффективный радиус, исследовались только в работе [1] и лишь в частном случае, названном "s-волновым". Даже этот частный случай не упоминается в современной монографии по теории рассеяния [2]. Поэтому исчерпывающий анализ двумерного ядерно-кулоновского рассеяния представляется актуальным и необходимым для расширения теории двумерного рассеяния.

Выполним такой анализ. В разделе 2 мы формулируем и обсуждаем исходную задачу Шредингера для радиальной волновой функции квантовой частицы. В разделе 3 мы сводим эту задачу к цепочке рекуррентных и энергонезависимых дифференциальных уравнений первого порядка с простыми начальными условиями. Эти цепочки исследуются в разделе 4 и используются в разделе 5 для вывода низкоэнергетических асимптотик функции эффективного радиуса, радиальных волновых функций, парциальных фаз и сечений рассеяния квантовой частицы. Итоги выполненных исследований подведены в заключении.

* Объединенный институт ядерных исследований, Дубна, Московская обл., Россия. E-mail: pupyshev@theor.jinr.ru 


\section{2. ИСХОДНАЯ РАДИАЛЬНАЯ ЗАДАЧА ШРЕДИНГЕРА}

Начнем с определений параметров, аргументов и потенциалов.

В плоскости $\mathcal{P}$ введем стандартным образом полярную систему координат с начальной точкой, совпадающей с силовым центром $O$. Положение квантовой частицы $p_{1}$ в плоскости $\mathcal{P}$ зададим ее полярными координатами $\mathbf{r}=(r, \varphi)$. Символами $m_{1}$, $z_{1}, E$ и $\mathbf{k}$ обозначим массу, кулоновский заряд, полную энергию и волновой вектор этой частицы. Считаем, что волновой вектор $\mathbf{k}_{0}$ ее начального состояния имеет нулевой азимутальный угол, а силовой центр $O$ обладает кулоновским зарядом $Z$. По определению заряды $z_{1}$ и $Z$ кратны заряду электрона. Пусть $\hbar-$ постоянная Планка. Положим

$$
\begin{gathered}
k=|\mathbf{k}| \equiv \sqrt{\frac{2 m_{1} E}{\hbar^{2}}}, \quad R \equiv \frac{\hbar^{2}}{2 m_{1} z_{1} Z}, \quad q \equiv k|R|, \\
\alpha \equiv \operatorname{sgn} R, \quad \eta \equiv \frac{1}{2 k R}=\frac{\alpha}{2 q}, \quad \rho \equiv k r=q \frac{r}{|R|}, \quad v \equiv \sqrt{8 \rho|\eta|} .
\end{gathered}
$$

Здесь и далее $q$ - безразмерное волновое число частицы $p_{1}, \eta$ - параметр Зоммерфельда, а $\rho$ и $v$ - безразмерные неотрицательные переменные.

Кулоновский потенциал определим равенством $V^{\mathrm{c}}(r)=z_{1} Z / r$. Придется различать два случая: кулоновского отталкивания $(\alpha=1)$ и кулоновского притяжения $(\alpha=-1)$. Для краткости записи используем символ Кронекера $\delta_{i, \alpha}$ и формулы с верхними и нижними знаками плюс и (или) минус. В таких формулах все верхние знаки берутся в случае $\alpha=1$, а все нижние - в случае $\alpha=-1$. Например, формула

$$
A^{ \pm}=a^{ \pm}+\left\{\begin{array}{l}
b \\
c
\end{array}\right\}
$$

означает, что $A^{+}=a^{+}+b$, если $\alpha=1$, и $A^{-}=a^{-}+c$, если $\alpha=-1$.

Считаем, что центральный короткодействующий потенциал $V^{\mathrm{s}}(r)$ зависит только от переменной $r$, принадлежит классу $C_{(0, \infty]}^{0}$ функций, непрерывных в области $r>0$, и при любом показателе $\beta>2$ удовлетворяет следующим условиям:

$$
\lim _{r \rightarrow 0} r^{2}\left|V^{\mathrm{s}}(r)\right|=0, \quad \lim _{r \rightarrow \infty} r^{\beta}\left|V^{\mathrm{s}}(r)\right|\left(\delta_{1, \alpha} e^{4 \sqrt{r /|R|}}+\delta_{-1, \alpha}\right)=0, \quad \alpha= \pm 1 .
$$

Поэтому безразмерный потенциал $V$, определенный формулами

$$
\left.V(v) \equiv \frac{2 m_{1} R^{2}}{\hbar^{2}} V^{\mathrm{s}}(r)\right|_{r=x|R|}, \quad x=2 \rho|\eta|=\frac{v^{2}}{4}, \quad r=\frac{v^{2}|R|}{4},
$$

подчиняется условиям

$$
\lim _{v \rightarrow 0} v^{4}|V(v)|=0, \quad V(v) \in C_{(0, \infty]}^{0}
$$

и при любом $\beta>2$ удовлетворяет соотношениям

$$
\lim _{v \rightarrow \infty} v^{2 \beta}|V(v)|\left(\delta_{1, \alpha} e^{2 v}+\delta_{-1, \alpha}\right)=0, \quad \alpha= \pm 1 .
$$

В некоторых случаях ограничения на допустимое поведение потенциалов $V^{\mathrm{s}}(r)$ и $V(v)$ при больших значениях аргументов можно ослабить. Все такие случаи мы укажем. Причину зависимости условия (2) от $\alpha$ мы объясним в конце п. 4.1. 
Перейдем к формулировке радиальной задачи Шредингера в случае суммарного потенциала $V^{\mathrm{c}}(r)+V^{\mathrm{s}}(r)$, не зависящего от угла $\varphi$. Как известно из теории двумерного рассеяния [2], [3], в этом случае двумерная задача Шредингера для волновой функции частицы $p_{1}$ допускает разделение переменных $r$ и $\varphi$ и сводится к бесконечной, но счетной $(2 \lambda=-1,1,3, \ldots)$ совокупности радиальных краевых задач Шредингера. В кулоновских переменных $\rho$ и $\eta$ каждая из таких задач представляет собой одномерное уравнение Шредингера

$$
\left[\partial_{\rho}^{2}-\frac{\lambda(\lambda+1)}{\rho^{2}}+1-\frac{2 \eta}{\rho}-4 \eta^{2} V(\sqrt{8 \rho|\eta|})\right] u_{\lambda}(\rho, \eta)=0, \quad \rho>0, \quad-\infty<\eta<\infty
$$

с условием

$$
\lim _{\rho \rightarrow 0} \frac{u_{\lambda}(\rho, \eta)}{F_{\lambda}(\rho, \eta)}=N(\eta)
$$

и условием

$$
u_{\lambda}(\rho, \eta)=\sin \left[\rho-\eta \ln 2 \rho-\frac{\pi \lambda}{2}+\delta_{\lambda}^{\mathrm{c}}(\eta)+\delta_{\lambda}(\eta)\right]+O\left(\rho^{-1}\right), \quad \rho \rightarrow \infty .
$$

В этих условиях $F_{\lambda}$ и $\delta_{\lambda}^{\mathrm{c}}-$ известные регулярная функция Кулона и кулоновская фаза [4]-[6], а нормировочный множитель $N$ и фаза $\delta_{\lambda}$ рассеяния потенциалом $V$ в кулоновском поле $2 \eta / \rho$ являются искомыми. Непрерывное в области $0 \leqslant \rho<\infty$ решение $u_{\lambda}$ задачи $(3)-(5)$ называется регулярной радиальной волновой функцией частицы $p_{1}$ в состоянии рассеяния $|q, \lambda\rangle$ с квантовыми числами $q$ и $\lambda$.

Радиальная краевая задача Шредингера (3)-(5) является исходной для наших исследований. Для полноты ее постановки следует пояснить строение сечения $\sigma_{\lambda}^{\mathrm{cs}}(\eta)$ рассеяния частицы $p_{1}$ в состоянии $|q, \lambda\rangle$. Такое сечение определяется известными формулами [3]

$$
\sigma_{\lambda}^{\mathrm{cs}}(\eta)=8 R \eta \varepsilon_{\lambda} \sin ^{2}\left[\delta_{\lambda}^{\mathrm{c}}(\eta)+\delta_{\lambda}(\eta)\right], \quad \varepsilon_{\lambda} \equiv 2-\delta_{-1,2 \lambda},
$$

и в силу тождества

$$
\sin ^{2}\left(\delta_{\lambda}^{\mathrm{c}}+\delta_{\lambda}\right) \equiv \sin ^{2} \delta_{\lambda}^{\mathrm{c}}+\sin ^{2} \delta_{\lambda}+2 \sin \delta_{\lambda}^{\mathrm{c}} \sin \delta_{\lambda} \cos \left(\delta_{\lambda}^{\mathrm{c}}+\delta_{\lambda}\right)
$$

является суммой слагаемых

$$
\begin{aligned}
\sigma_{\lambda}^{\mathrm{c}}(\eta) & \equiv 8 R \eta \varepsilon_{\lambda} \sin ^{2} \delta_{\lambda}^{\mathrm{c}}(\eta), \\
\sigma_{\lambda}^{\mathrm{int}}(\eta) & \equiv 16 R \eta \varepsilon_{\lambda} \sin \delta_{\lambda}^{\mathrm{c}}(\eta) \sin \delta_{\lambda}(\eta) \cos \left[\delta_{\lambda}^{\mathrm{c}}(\eta)+\delta_{\lambda}(\eta)\right]
\end{aligned}
$$

и слагаемого

$$
\sigma_{\lambda}(\eta) \equiv 8 R \eta \varepsilon_{\lambda} \sin ^{2} \delta_{\lambda}(\eta)=\frac{\sigma_{\lambda}^{\mathrm{u}}(\eta)}{\operatorname{ctg}^{2} \delta_{\lambda}(\eta)+1}, \quad \text { где } \quad \sigma_{\lambda}^{\mathrm{u}}(\eta) \equiv 8 R \eta \varepsilon_{\lambda} .
$$

Слагаемое $\sigma_{\lambda}^{\mathrm{c}}$ - сечение рассеяния одним кулоновским потенциалом. Слагаемое $\sigma_{\lambda}^{\text {int }}$ описывает вклад от интерференции рассеяния кулоновским потенциалом и потенциалом $V$. Слагаемое $\sigma_{\lambda}-$ сечение рассеяния этим потенциалом в кулоновском поле. При любом $\eta$ такое сечение не превышает своего унитарного предела $\sigma_{\lambda}^{\mathrm{u}}$. Как и в ядерной физике, функции $\delta_{\lambda}(\eta)$ и $\sigma_{\lambda}(\eta)$ мы называем парциальными ядернокулоновскими фазой и сечением. 
Обсудим задачу Шредингера (3)-(5). Для этого сначала напомним определение предела низких энергий. Пределом низких энергий называется предел $q \rightarrow+0$ при фиксированных значениях кулоновского параметра $R$ и квантового числа $\lambda$. В этом пределе $|\eta| \rightarrow \infty$. В случае потенциала $V(v) \equiv 0$, где $v=\sqrt{8 \rho|\eta|}$, уравнению (3) удовлетворяют линейно независимые волновые функции Кулона $F_{\lambda}(\rho, \eta)$ и $G_{\lambda}(\rho, \eta)$ полуцелого порядка $\lambda=-1 / 2,1 / 2,3 / 2, \ldots$, детально исследованные в работах [4]-[6]. Как доказано в работе [7], при любых допустимых значениях $\lambda$ и $\eta$ и при условиях

$$
\lim _{v \rightarrow 0} v^{4} V(v)=0, \quad V(v) \in C_{(0, \infty]}^{0}, \quad \lim _{v \rightarrow \infty} v^{4} V(v)=0
$$

задача (3)-(5) имеет единственное, причем непрерывное в области $0 \leqslant \rho<\infty$, решение $u_{\lambda}$. Короткодействующий потенциал $V$ по определению удовлетворяет условиям (1) и (2), а значит, и условиям (8). Вывод низкоэнергетических (при $q \rightarrow 0$, $|\eta| \rightarrow \infty)$ приближений функций $\delta_{\lambda}(\eta), \sigma_{\lambda}(\eta), N(\eta)$ и $u_{\lambda}(\rho, \eta)$ в рамках задачи $(3)-(5)$ представляет собой довольно сложную проблему. Для ее наиболее простого решения в разделах 3 и 4 предлагается специальный метод, асимптотический в пределе $q \rightarrow 0$. Первый этап этого метода заключается в переформулировке задачи (3)-(5) и реализуется в следующем разделе.

\section{3. РЕДУКЦИЯ РАДИАЛЬНОЙ ЗАДАЧИ ШРЕДИНГЕРА}

В настоящем разделе проводится редукция радиальной задачи Шредингера (3)-(5) к системам линейных дифференциальных уравнений первого порядка, наиболее удобных для вывода и анализа низкоэнергетических асимптотик фазы $\delta_{\lambda}$, сечения $\sigma_{\lambda}$ и волновой функции $u_{\lambda}$. Такая редукция реализуется в три этапа. Каждому из них посвящен отдельный пункт.

3.1. Амплитудные функции. Кратко изложим и обсудим метод амплитудных функций, предложенный в работе [7]. В этой работе в отличие от настоящей использовался потенциал $V(x)$, где $x \equiv v^{2} / 4=2 \rho|\eta|$.

В методе амплитудных функций кулоновские функции $F_{\lambda}$ и $G_{\lambda}$ считаются известными, а амплитудные функции $c$ и $s$ - искомыми. Решение $u_{\lambda}$ задачи Шредингера (3)-(5) представляется как

$$
u_{\lambda}(\rho, \eta)=N(\eta) U(\rho, \eta), \quad U(\rho, \eta) \equiv c(\rho, \eta) F_{\lambda}(\rho, \eta)+s(\rho, \eta) G_{\lambda}(\rho, \eta) .
$$

Подстановкой (9) уравнение Шредингера (3) с начальным условием (4) сводится к задаче Коши для амплитудных функций $c$ и $s$ на полуоси $\rho \geqslant 0$, а именно к недиагональной системе двух линейных дифференциальных уравнений первого порядка

$$
\begin{aligned}
& \partial_{\rho} c(\rho, \eta)=4 \eta^{2} V(\sqrt{8 \rho|\eta|})\left[c(\rho, \eta) F_{\lambda}(\rho, \eta)+s(\rho, \eta) G_{\lambda}(\rho, \eta)\right] G_{\lambda}(\rho, \eta), \\
& \partial_{\rho} s(\rho, \eta)=-4 \eta^{2} V(\sqrt{8 \rho|\eta|})\left[c(\rho, \eta) F_{\lambda}(\rho, \eta)+s(\rho, \eta) G_{\lambda}(\rho, \eta)\right] F_{\lambda}(\rho, \eta)
\end{aligned}
$$

с начальными условиями

$$
\left.\left.c(\rho, \eta)\right|_{\rho=0}=1, \quad s(\rho, \eta)\right)\left.\right|_{\rho=0}=0 .
$$

В работе [7] доказано следующее утверждение, справедливое и в рассматриваемом случае короткодействующего потенциала $V$. 
Теорема 1. Если потенииал $V(v), v=\sqrt{8 \rho|\eta|}$, удовлетворяет условиям (8), то при любом $\lambda \geqslant-1 / 2$ и любом $\eta \in(-\infty, \infty)$ задача Коши (10), (11) однозначно разрешима в классе функиий, непрерывных на всей полуоси $\rho \geqslant 0$, а компоненты решения $\{c, s\}$ этой задачи не имеют общих нулей.

Как показано в той же работе [7], в силу теоремы 1 представления множителя $N$, фазы $\delta_{\lambda}$ и сечения $\sigma_{\lambda}$ через амплитудные функции $c$ и $s$ являются однозначными и непрерывными. Приведем такие представления: множитель $N$ определяется формулами

$$
N(\eta)=\lim _{\rho \rightarrow \infty} N(\rho, \eta), \quad N(\rho, \eta) \equiv\left[c^{2}(\rho, \eta)+s^{2}(\rho, \eta)\right]^{-1 / 2},
$$

тангенс фазы рассеяния $\delta_{\lambda}-$ формулами

$$
\operatorname{tg} \delta_{\lambda}(\eta)=\lim _{\rho \rightarrow \infty} \operatorname{tg} \delta_{\lambda}(\rho, \eta), \quad \operatorname{tg} \delta_{\lambda}(\rho, \eta) \equiv \frac{s(\rho, \eta)}{c(\rho, \eta)}
$$

а сечение (7) вычисляется как

$$
\sigma_{\lambda}(\eta)=\lim _{\rho \rightarrow \infty} \sigma_{\lambda}(\rho, \eta), \quad \sigma_{\lambda}(\rho, \eta) \equiv \sigma_{\lambda}^{\mathrm{u}}(\eta)[s(\rho, \eta) N(\rho, \eta)]^{2} .
$$

В представленном выше методе амплитудных функций [7] для полного решения краевой задачи Шредингера (3)-(5) необходимо сначала найти решение $\{c, s\}$ задачи Коши $(10),(11)$, затем определить множитель $N$, фазу $\delta_{\lambda}$ и сечение $\sigma_{\lambda}$ по формулам (12)-(14) и, наконец, вычислить радиальную волновую функцию $u_{\lambda}$ по формулам (9).

Выявим причины, которые существенно затрудняют численный анализ задачи Коши $(10),(11)$ и последующее вычисление функций $\delta_{\lambda}, \sigma_{\lambda}$ и $u_{\lambda}$ в пределе низких энергий $q \rightarrow 0,|\eta| \rightarrow \infty$.

При малых значениях аргумента $\rho \rightarrow 0$ функции Кулона имеют асимптотики [4]

$$
F_{\lambda}(\rho, \eta) \sim C_{\lambda}(\eta) \rho^{\lambda+1}, \quad G_{\lambda}(\rho, \eta) \sim \frac{1}{C_{\lambda}(\eta) \rho^{\lambda}} \begin{cases}-\ln \rho, & 2 \lambda=-1 \\ (2 \lambda+1)^{-1}, & 2 \lambda>-1\end{cases}
$$

в которых неаналитическая функция $C_{\lambda}(\eta)$ определяется формулой

$$
C_{\lambda}(\eta) \equiv 2^{\lambda} \frac{|\Gamma(\lambda+1+\imath \eta)|}{\Gamma(2 \lambda+2)} e^{-\pi \eta / 2}
$$

Поэтому квадрат этой функции имеет асимптотику

$$
C_{\lambda}^{2}( \pm|\eta|) \sim \frac{\pi}{(\nu !)^{2}} q^{-\nu}\left\{\begin{array}{c}
e^{-\pi / q} \\
1
\end{array}\right\}, \quad \nu=2 \lambda+1, \quad \frac{q}{|\lambda|} \rightarrow 0 .
$$

Следовательно, при $q \rightarrow 0$ в случае кулоновского отталкивания $(\alpha \equiv \operatorname{sgn} \eta=1)$ функция $C_{\lambda}$ быстро убывает, а в случае кулоновского притяжения $(\alpha=-1)$ сходится к числу $\sqrt{\pi}$, если $2 \lambda=-1$, и возрастает, как $O\left(q^{-\lambda-1 / 2}\right)$, если $2 \lambda>-1$.

Вследствие свойств (15)-(17) кулоновских функций при $q \rightarrow 0$ и $\rho \ll 1$ правые части уравнений $(10)$ содержат как быстро убывающий множитель $F_{\lambda}^{2}$, так и быстро 
возрастающий множитель $G_{\lambda}^{2}$. Поэтому при уменьшении волнового числа $q$ численное интегрирование таких уравнений в области $\rho \ll 1$ становится все более сложной задачей.

В пределе $\rho \rightarrow \infty$ функции Кулона имеют асимптотики [4]

$$
\begin{aligned}
& F_{\lambda}(\rho, \eta) \sim \sin \chi_{\lambda}(\rho, \eta), \\
& G_{\lambda}(\rho, \eta) \sim \cos \chi_{\lambda}(\rho, \eta), \quad \chi_{\lambda}(\rho, \eta) \equiv \rho-\eta \ln 2 \rho-\frac{\pi \lambda}{2}+\delta_{\lambda}^{\mathrm{c}}(\eta),
\end{aligned}
$$

в которых модуль фазы $\delta_{\lambda}^{\mathrm{c}}(\eta)$ неограниченно возрастает, если $q /|\lambda| \rightarrow 0$ :

$$
\delta_{\lambda}^{\mathrm{c}}(\eta) \sim \frac{\eta}{2} \ln \left[(\lambda+1)^{2}+\eta^{2}\right]-\eta+\left(\lambda+\frac{1}{2}\right) \operatorname{arctg} \frac{\eta}{\lambda+1} .
$$

Следовательно, в пределе $q \rightarrow 0$ в области $\rho \gg 1$ функции $F_{\lambda}^{2}, F_{\lambda} G_{\lambda}$ и $G_{\lambda}^{2}$ быстро осциллируют. Поэтому следует ожидать, что правые части уравнений (10), а значит, и производные $\partial_{\rho} c, \partial_{\rho} s$, и функции $c, s$ будут быстро осциллировать. Анализ быстро осциллирующих функций - одна из наиболее трудных задач численного интегрирования дифференциальных уравнений.

Функция $G_{\lambda}$ записывается как [4]

$$
G_{\lambda}(\rho, \eta)=-\frac{h^{\mathrm{c}}(\eta)}{C_{-1 / 2}^{2}(\eta)} F_{\lambda}(\rho, \eta)+\frac{1}{q^{\lambda} C_{\lambda}(\eta)} \Theta_{\lambda}(\rho, \eta) .
$$

В этой сумме $\Theta_{\lambda}$ - четная функция аргумента $q$, а $h^{\mathrm{c}}(\eta)$ - неаналитическая и четная функция переменой $\eta$. Эта функция содержит в качестве слагаемого вещественную часть $\Psi$-функции, а в пределе $q \rightarrow 0$ аппроксимируется полиномом по переменной $q^{2}$ :

$$
\begin{aligned}
h^{\mathrm{c}}(\eta) & \equiv \operatorname{Re} \Psi\left(\frac{1}{2}+\imath \eta\right)-\ln |\eta|, \\
h^{\mathrm{c}}(\eta) & =-\frac{1}{6} q^{2}-\frac{7}{60} q^{4}+O\left(q^{6}\right), \quad \eta=\frac{\alpha}{2 q}, \quad q \rightarrow 0 .
\end{aligned}
$$

Правые части обсуждаемых уравнений (10) содержат и функцию $F_{\lambda}$, и функцию $G_{\lambda}$, удовлетворяющие соотношениям (15) и (19). Следовательно, по крайней мере одна из амплитудных функций $c$ или $s$ должна содержать неаналитические функции $C_{\lambda}(\eta)$ и $h^{\mathrm{c}}(\eta)$ в качестве множителей или слагаемых. В силу представлений (9) и (12)-(14) этим же свойством должны обладать функции $U, N, u_{\lambda}, \delta_{\lambda}$ и $\sigma_{\lambda}$. Поэтому вывод явных низкоэнергетических асимптотик этих функций представляется довольно сложным и требует особого подхода.

Из проведенного выше обсуждения задачи (10), (11) следует, что ее необходимо свести к альтернативной задаче Коши, наиболее удобной для численного анализа в пределе низких энергий, и попутно найти представления амплитудных функций $c$ и $s$, в которых неаналитические функции $C_{\lambda}$ и $h^{\mathrm{c}}$ выделены в явном виде. Вывод и обсуждение таких представлений мы проведем ниже.

3.2. Редуцированные амплитудные функции. Мы предлагаем новую формулировку метода амплитудных функций, наиболее адаптированную для анализа 
радиальной задачи Шредингера (3)-(5) в пределе низких энергий. Основными в такой формулировке будут редуцированные амплитудные функции $c^{ \pm}$и $s^{ \pm}$.

Используем известные представления [4] функций $F_{\lambda}$ и $\Theta_{\lambda}$ :

$$
\begin{aligned}
& F_{\lambda}(\rho, \pm|\eta|)=q^{\lambda+1} C_{\lambda}(\eta) \nu ! v f_{\lambda}^{ \pm}(v, q), \quad v=\sqrt{8 \rho|\eta|}, \quad \rho \geqslant 0, \quad \nu \equiv 2 \lambda+1 \\
& \Theta_{\lambda}(\rho, \pm|\eta|)=\frac{v}{\nu !} \theta_{\lambda}^{ \pm}(v, q),
\end{aligned}
$$

Положим

$$
\begin{aligned}
& s(\rho, \pm|\eta|)=(\nu !)^{2} q^{2 \lambda+1} C_{\lambda}^{2}( \pm|\eta|) s^{ \pm}(v, q), \\
& c(\rho, \pm|\eta|)=c^{ \pm}(v, q)+\frac{h^{\mathrm{c}}( \pm|\eta|)}{C_{-1 / 2}^{2}( \pm|\eta|)} s(\rho, \pm|\eta|)=c^{ \pm}(v, q)+h(q) s^{ \pm}(v, q),
\end{aligned}
$$

где четная функция $h(q)$ имеет вид произведения

$$
h(q) \equiv(\nu !)^{2} h^{\mathrm{c}}(\eta) P_{\lambda}\left(q^{2}\right), \quad P_{\lambda}\left(q^{2}\right) \equiv q^{2 \lambda+1} \frac{C_{\lambda}^{2}(\eta)}{C_{-1 / 2}^{2}(\eta)},
$$

в котором $h^{\text {c }}$ - функция $(20)$, а $P_{\lambda}$ - известный полином [4] степени $2 \lambda+1$ :

$$
P_{\lambda}\left(q^{2}\right)=\frac{1}{(\nu !)^{2}}\left\{1+\frac{2}{3} \lambda(\lambda+1)(2 \lambda+1) q^{2}\left[1+\frac{1}{15}(\lambda-1)(2 \lambda-1)(5 \lambda+6) q^{2}+\cdots\right]\right\} .
$$

Поэтому $h(q)=h^{\mathrm{c}}(\eta)$ в случае $2 \lambda=-1$, а при условии $2 \lambda>-1$ благодаря формулам (20)

$$
h(q)=-\frac{1}{6} q^{2}-\frac{1}{3}\left[\frac{7}{20}+\frac{1}{3} \lambda(\lambda+1)(2 \lambda+1)\right] q^{4}+O\left(q^{6}\right), \quad q \rightarrow 0 .
$$

В определении (9) функции $U$ и в задаче $(10),(11)$ заменим функцию $G_{\lambda}$ суммой (19), функции $F_{\lambda}$ и $\Theta_{\lambda}$ представим в виде (21), а функции $c$ и $s-$ в виде $(22)$. В полученных соотношениях приведем подробные слагаемые. В итоге для функции $U$ выведем представление

$$
\begin{gathered}
U(\rho, \pm|\eta|)=\nu ! q^{\lambda+1} C_{\lambda}( \pm|\eta|) U^{ \pm}(v, q), \\
U^{ \pm}(v, q) \equiv v\left\{c^{ \pm}(v, q) f_{\lambda}^{ \pm}(v, q)+s^{ \pm}(v, q) \theta_{\lambda}^{ \pm}(v, q)\right\},
\end{gathered}
$$

в котором функции $c^{ \pm}$и $s^{ \pm}$в области $v>0$ подчиняются системе уравнений

$$
\begin{aligned}
& \partial_{v} c^{ \pm}(v, q)=v^{3} V(v)\left[c^{ \pm}(v, q) f_{\lambda}^{ \pm}(v, q)+s^{ \pm}(v, q) \theta_{\lambda}^{ \pm}(v, q)\right] \theta_{\lambda}^{ \pm}(v, q), \\
& \partial_{v} s^{ \pm}(v, q)=-v^{3} V(v)\left[c^{ \pm}(v, q) f_{\lambda}^{ \pm}(v, q)+s_{\lambda}^{ \pm}(v, q) \theta_{\lambda}^{ \pm}(v, q)\right] f_{\lambda}^{ \pm}(v, q)
\end{aligned}
$$

с начальными условиями

$$
\left.c^{ \pm}(v, q)\right|_{v=0}=1,\left.\quad s^{ \pm}(v, q)\right|_{v=0}=0 .
$$

Вследствие теоремы 1 и однозначности представлений (22) функций $c$ и $s$ через функции $c^{ \pm}$и $s^{ \pm}$верно следующее важное утверждение. 
Теорема 2. Если потенциал $V$ удовлетворяет условиям (8), то при любом $\lambda \geqslant-1 / 2$ и любом $q \geqslant 0$ задача Коши (27), (28) имеет единственное, причем непрерьвное на всей полуоси $v \geqslant 0$, решение $\left\{c^{ \pm}, s^{ \pm}\right\}$.

Завершим редукцию радиальной задачи Шредингера (3)-(5) выводом специальных представлений функций $N, \delta_{\lambda}, \sigma_{\lambda}$ и $u_{\lambda}$, в которых все неаналитические функции аргумента $q$ выделены в явном виде. Для этого в равенствах (12)-(14) выразим функции $c$ и $s$ через функции $c^{ \pm}$и $s^{ \pm}$по формулам (22). В результате получим соотношения

$$
\begin{gathered}
N( \pm|\eta|)=\lim _{v \rightarrow \infty} N^{ \pm}(v, q) \\
N^{ \pm}(v, q) \equiv\left\{\left[c^{ \pm}(v, q)+h(q) s^{ \pm}(v, q)\right]^{2}+\left[(\nu !)^{2} q^{2 \lambda+1} C_{\lambda}^{2}( \pm|\eta|) s^{ \pm}(v, q)\right]^{2}\right\}^{-1 / 2},
\end{gathered}
$$

а также формулы

$$
\begin{gathered}
\operatorname{tg} \delta_{\lambda}( \pm|\eta|)=\lim _{v \rightarrow \infty} \operatorname{tg} \delta_{\lambda}^{ \pm}(v, q) \\
\operatorname{tg} \delta_{\lambda}^{ \pm}(v, q) \equiv(\nu !)^{2} q^{2 \lambda+1} C_{\lambda}^{2}( \pm|\eta|) \frac{s^{ \pm}(v, q)}{\left[c^{ \pm}(v, q)+h(q) s^{ \pm}(v, q)\right]}
\end{gathered}
$$

и представления

$$
\begin{gathered}
\sigma_{\lambda}( \pm|\eta|)=\sigma_{\lambda}^{ \pm}(q) \equiv \lim _{v \rightarrow \infty} \sigma_{\lambda}^{ \pm}(v, q) \\
\sigma_{\lambda}^{ \pm}(v, q) \equiv 4 \varepsilon_{\lambda}|R| q\left[\nu ! q^{\lambda} C_{\lambda}( \pm|\eta|)\right]^{4}\left[s^{ \pm}(v, q) N^{ \pm}(v, q)\right]^{2} .
\end{gathered}
$$

Используя соотношения $(9),(26)$ и $(29)$, представим волновую функцию $u_{\lambda}$ в виде

$$
\begin{gathered}
u_{\lambda}(\rho, \pm|\eta|)=\nu ! q^{\lambda+1} C_{\lambda}( \pm|\eta|) N( \pm|\eta|) U^{ \pm}(v, q), \\
U^{ \pm}(v, q)=v\left[c^{ \pm}(v, q) f_{\lambda}^{ \pm}(v, q)+s^{ \pm}(v, q) \theta_{\lambda}^{ \pm}(v, q)\right] .
\end{gathered}
$$

Итак, для полного решения исходной задачи (3)-(5) необходимо сначала решить задачу (27), (28), затем найти множитель $N$, фазу $\delta_{\lambda}$ и сечение $\sigma_{\lambda}$ по формулам (29)-(31) и, наконец, вычислить функцию $u_{\lambda}$ по формулам (32). В силу теоремы 2 при условиях (8) такая функция будет единственным и непрерывным решением задачи (3)-(5).

Обсудим преимущества новых уравнений (27) по сравнению с исходными уравнениями (10). Вследствие представлений (21) уравнения (27) вместо функций Кулона $F_{\lambda}$ и $G_{\lambda}$ содержат $f_{\lambda}^{ \pm}$и $\theta_{\lambda}^{ \pm}$. Как известно [6], в области $0 \leqslant v<\infty, 0 \leqslant q<\infty$ эти функции являются равномерно сходящимися рядами по четным степеням аргумента $q$ :

$$
f_{\lambda}^{ \pm}(v, q)=\sum_{n=0}^{\infty} q^{2 n} f_{\lambda n}^{ \pm}(v), \quad \theta_{\lambda}^{ \pm}(v, q)=\sum_{n=0}^{\infty} q^{2 n} \theta_{\lambda n}^{ \pm}(q) .
$$

Поэтому обе компоненты решения $\left\{c^{ \pm}, s^{ \pm}\right\}$задачи Коши (27), (28) в отличие от функций $c$ и $s$ не содержат в качестве множителей или слагаемых никаких неаналитических функций переменной $q$. Более того, следует ожидать, что в области $0 \leqslant v<\infty, 0 \leqslant q<\infty$ функции $c^{ \pm}$и $s^{ \pm}$будут равномерно сходящимися рядами того же типа, что и ряды (33):

$$
c^{ \pm}(v, q)=\sum_{n=0}^{\infty} q^{2 n} c_{n}^{ \pm}(v), \quad s^{ \pm}(v, q)=\sum_{n=0}^{\infty} q^{2 n} s_{n}^{ \pm}(v) .
$$


Следовательно, функции $c^{ \pm}$и $s^{ \pm}$устроены более просто, чем породившие их функции $c$ и $s$. Благодаря указанным выше свойствам функций $f_{\lambda}^{ \pm}, \theta_{\lambda}^{ \pm}, c^{ \pm}$и $s^{ \pm}$численное интегрирование уравнений (27) в пределе низких энергий - довольно простая задача.

\section{3. Уравнения для компонент редуцированных амплитудных функ-} ций. Выведем и обсудим задачи Коши для искомых компонент $c_{n}^{ \pm}$и $s_{n}^{ \pm}$рядов (34). Для этого в системе уравнений (27) и в ее начальных условиях (28) представим функции $f_{\lambda}^{ \pm}$и $\theta_{\lambda}^{ \pm}$известными рядами (33), а редуцированные амплитудные функции $c^{ \pm}$и $s^{ \pm}-$искомыми рядами (34). Получившиеся равенства запишем в виде бесконечных полиномов по четным степеням параметра $q$. Благодаря линейной независимости системы $\left\{q^{2 n}\right\}_{n=0}^{\infty}$ степенных функций эти полиномы тождественно равны нулю тогда и только тогда, когда равны нулю все их "коэффициенты", зависящие лишь от переменной $v$. Применив это правило, для каждого значения $n=0,1, \ldots$ на полуоси $v>0$ получим искомую систему двух уравнений

$$
\begin{aligned}
& \partial_{v} c_{n}^{ \pm}=v^{3} V\left[c_{n}^{ \pm} f_{\lambda 0}^{ \pm}+s_{n}^{ \pm} \theta_{\lambda 0}^{ \pm}\right] \theta_{\lambda 0}^{ \pm}+v^{3} V \sum_{k=0}^{n-1} \sum_{\ell+p=n-k}\left[c_{k}^{ \pm} f_{\lambda \ell}^{ \pm}+s_{i}^{ \pm} \theta_{\lambda \ell}^{ \pm}\right] \theta_{\lambda p}^{ \pm}, \\
& \partial_{v} s_{n}^{ \pm}=-v^{3} V\left[c_{n}^{ \pm} f_{\lambda 0}^{ \pm}+s_{n}^{ \pm} \theta_{\lambda 0}^{ \pm}\right] f_{\lambda 0}^{ \pm}-v^{3} V \sum_{k=0}^{n-1} \sum_{\ell+p=n-k}\left[c_{k}^{ \pm} f_{\lambda \ell}^{ \pm}+s_{i}^{ \pm} \theta_{\lambda \ell}^{ \pm}\right] f_{\lambda p}^{ \pm}
\end{aligned}
$$

с начальными условиями

$$
\left.c_{0}^{ \pm}(v)\right|_{v=0}=\delta_{0, n},\left.\quad s_{n}^{ \pm}(v)\right|_{v=0}=0 .
$$

В уравнениях (35) при $n=0$ суммы с верхним индексом $n-1$ по определению полагаются равными нулю.

Обсудим строение цепочки задач Коши (35), (36). В отличие от исходной задачи $(27),(28)$ все (для $n=0,1, \ldots)$ задачи $(35),(36)$ не содержат волновое число $q$ и поэтому называются энергонезависимыми. Первая (для $n=0$ ) система (35) является однородной, а любая другая (для $n>0)$ - неоднородной и содержит в своей правой части компоненты решений $\left\{c_{m}^{ \pm}, s_{m}^{ \pm}\right\}$всех предыдущих (для $m=0,1, \ldots, n-1$ ) систем (35). Следовательно, цепочка задач (35), (36) является рекуррентной по индексу $n$.

Заметим, что анализ системы (35) методом последовательных приближений Пикара-Линделёфа [8] затрудняется двумя обстоятельствами: при любом $n$ система недиагональная, а мажорантные оценки для $f_{\lambda 0}^{ \pm}(w) \theta_{\lambda 0}^{ \pm}(v), w>v$, неизвестны.

Чтобы преодолеть оба указанных затруднения, для каждого $n=0,1, \ldots$ сведем задачу Коши (35), (36) к диагональной системе интегральных уравнений для функций $y_{1 n}^{ \pm}$и $y_{2 n}^{ \pm}$. Для этого сначала определим интеграл $b^{ \pm}$и функции $p_{1}^{ \pm}$и $p_{2}^{ \pm}$как

$$
b^{ \pm}(v) \equiv \int_{0}^{v} t^{3} V(t) f_{\lambda 0}^{ \pm}(t) \theta_{\lambda 0}^{ \pm}(t) d t
$$

И

$$
p_{1}^{ \pm}(v) \equiv v^{3} V(v)\left[\theta_{\lambda 0}^{ \pm}(v)\right]^{2} e^{-2 b^{ \pm}(v)}, \quad p_{2}^{ \pm}(v) \equiv-v^{3} V(v)\left[f_{\lambda 0}^{ \pm}(v)\right]^{2} e^{+2 b^{ \pm}(v)} .
$$

Затем подстановкой

$$
c_{n}^{ \pm}(v)=y_{1 n}^{ \pm}(v) e^{b^{ \pm}(v)}, \quad s_{n}^{ \pm}(v)=y_{2 n}^{ \pm}(v) e^{-b^{ \pm}(v)}
$$


сведем исходную задачу Коши (35), (36) к системе дифференциальных уравнений

$$
\partial_{v} y_{i n}^{ \pm}(v)=p_{i}^{ \pm}(v) y_{j n}^{ \pm}(v)+a_{i n}^{ \pm}(v), \quad i=1,2, \quad j=1+\delta_{1, i}, \quad v>0,
$$

с начальными условиями $y_{i n}^{ \pm}(v)=\delta_{0, n} \delta_{1, i}, i=1,2$, в точке $v=0$. Функции $a_{1 n}^{ \pm}$ и $a_{2 n}^{ \pm}$тождественно равны нулю, если $n=0$, а при $n>0$ являются конечными двукратными суммами. Опустив для краткости аргумент $v$, запишем эти суммы в виде

$$
\begin{aligned}
& a_{1 n}^{ \pm} \equiv v^{3} V \sum_{k=0}^{n-1} \sum_{\ell+p=n-k}\left\{y_{1 k}^{ \pm} f_{\lambda \ell}^{ \pm}+y_{2 k}^{ \pm} \theta_{\lambda \ell}^{ \pm} e^{-2 b^{ \pm}}\right\} \theta_{\lambda p}^{ \pm}, \\
& a_{2 n}^{ \pm} \equiv-v^{3} V \sum_{k=0}^{n-1} \sum_{\ell+p=n-k}\left\{y_{1 k}^{ \pm} f_{\lambda \ell}^{ \pm} e^{2 b^{ \pm}}+y_{2 k}^{ \pm} \theta_{\lambda \ell}^{ \pm}\right\} f_{\lambda p}^{ \pm} .
\end{aligned}
$$

Теперь представим систему дифференциальных уравнений (40) в виде недиагональной системы двух интегральных уравнений (здесь $i=1,2$ и $j=1+\delta_{1, i}$ ):

$$
y_{i n}^{ \pm}(v)=d_{i n}^{ \pm}(v)+\int_{0}^{v} p_{i}^{ \pm}(t) y_{j n}^{ \pm}(t) d t, \quad d_{i n}^{ \pm}(v)=\delta_{0, n} \delta_{1, i}+\int_{0}^{v} a_{i n}^{ \pm}(t) d t .
$$

Наконец, с помощью итерации этих уравнений выведем искомую диагональную систему:

$$
\begin{aligned}
& y_{i n}^{ \pm}(v)=r_{i n}^{ \pm}(v)+\int_{0}^{v} p_{i}^{ \pm}\left(t_{1}\right) d t_{1} \int_{0}^{t_{1}} p_{j}^{ \pm}(t) y_{i n}^{ \pm}(t) d t, \\
& r_{i n}^{ \pm}(v)=d_{i n}^{ \pm}(v)+\int_{0}^{v} p_{i}^{ \pm}(t) d_{j n}^{ \pm}(t) d t .
\end{aligned}
$$

Первое (при $i=1, j=2$ ) уравнение этой системы не содержит функцию $y_{2 n}^{ \pm}$, а второе (при $i=2, j=1$ ) не содержит функцию $y_{1 n}^{ \pm}$. Поэтому эти уравнения можно исследовать независимо друг от друга. В этом заключается первое и неоспоримое преимущество системы (43) по сравнению с недиагональной системой (35). Второе преимущество состоит в том, что достаточно решить только первое из уравнений системы (43), а затем вычислить $y_{2 n}^{ \pm}$как интегральный образ (42) уже найденной функции $y_{1 n}^{ \pm}$. Еще одно преимущество системы (43) порождается строением сумм (41): при любом $n$ эти суммы, а значит, и интегралы $d_{i n}^{ \pm}$и $r_{i n}^{ \pm}$, стоящие в уравнениях (42) и (43), содержат искомые функции $y_{1 k}^{ \pm}$и $y_{2 k}^{ \pm}$с номерами $k \leqslant n-1$. Поэтому системы двух уравнений (43) образуют рекуррентную по индексу $n$ цепочку систем, которые можно исследовать в порядке возрастания этого индекса.

В следующем разделе мы используем все преимущества систем (43).

\section{4. СВОЙСТВА КОМПОНЕНТ РЕДУЦИРОВАННЫХ АМПЛИТУДНЫХ ФУНКЦИЙ}

Следующий этап построения предлагаемого асимптотического метода - анализ свойств компонент $c_{n}^{ \pm}$и $s_{n}^{ \pm}$. Наша очередная задача - доказать существование и единственность всех таких компонент в случае, когда все вспомогательные функции $d_{i n}^{ \pm}, r_{i n}^{ \pm}$и $y_{i n}^{ \pm}, i=1,2, n=0,1, \ldots$, непрерывны на полуоси $v \geqslant 0$ и, следовательно, в обоих пределах $v \rightarrow 0$ и $v \rightarrow \infty$ принимают вполне определенные конечные значения. 
Для решения поставленной задачи используем представления (39) компонент $c_{n}^{ \pm}$ и $s_{n}^{ \pm}$через функции $y_{1 n}^{ \pm}$и $y_{2 n}^{ \pm}$, интегральные соотношения (42) и уравнения (43). В п. 4.1 приведены и обсуждаются известные представления компонент $f_{\lambda n}^{ \pm}$и $\theta_{\lambda n}^{ \pm}$ разложений (33). В п. 4.2 представления компонент $f_{\lambda 0}^{ \pm}$и $\theta_{\lambda 0}^{ \pm}$используются для того, чтобы доказать вспомогательные утверждения, сформулированные в виде лемм 1 и 2. В п. 4.3 воспользуемся этими леммами для доказательства теорем существования и единственности.

\section{1. Компоненты разложений функций Кулона и ограничения на по-} тенциал. Как доказано в работе [6], компоненты $f_{\lambda n}^{ \pm}$и $\theta_{\lambda n}^{ \pm}$рядов (33) определяются формулами

$$
\begin{gathered}
f_{\lambda n}^{ \pm}(v) \equiv \frac{(-1)^{n}}{2^{3 n+1}} \frac{1}{n !} \sum_{s=2 n}^{3 n} b_{n s} v^{s}\left\{\begin{array}{c}
(-1)^{s} I_{\nu+s}(v) \\
J_{\nu+s}(v)
\end{array}\right\}, \quad \nu \equiv 2 \lambda+1, \\
\theta_{\lambda n}^{ \pm}(v) \equiv\left(-\frac{1}{8}\right)^{n} \frac{1}{n !} \sum_{s=2 n}^{3 n}(-1)^{s} b_{n s} v^{s}\left\{\begin{array}{c}
\alpha_{n s}^{+} I_{\nu+s}(v)+\beta_{n s}^{+} K_{\nu+s}(v) \\
(-1)^{s} \alpha_{n s}^{-} J_{\nu+s}(v)+\beta_{n s}^{-}(-\pi / 2) Y_{\nu+s}(v)
\end{array}\right\} .
\end{gathered}
$$

В этих формулах $I_{\mu}, J_{\mu}$ и $K_{\mu}, Y_{\mu}$ - функции Бесселя первого и второго рода, а $b_{n s}^{ \pm}$, $\alpha_{n s}^{ \pm}$и $\beta_{n s}^{ \pm}-$коэффициенты. Оптимальные способы их вычисления предложены в той же работе [6]. Согласно этому вычислению компоненты $f_{\lambda 0}^{ \pm}$и $\theta_{\lambda 0}^{ \pm}$пропорциональны функциям Бесселя:

$$
f_{\lambda 0}^{ \pm}(v)=\frac{1}{2}\left\{\begin{array}{l}
I_{\nu}(v) \\
J_{\nu}(v)
\end{array}\right\}, \quad \theta_{\lambda 0}^{ \pm}(v)=\left\{\begin{array}{c}
K_{\nu}(v) \\
(-\pi / 2) Y_{\nu}(v)
\end{array}\right\}
$$

а компоненты $f_{\lambda n}^{ \pm}$и $\theta_{\lambda n}^{ \pm}, n>0$, устроены более сложно. Так, компоненты $\theta_{\lambda n}^{+}$и $\theta_{\lambda n}^{-}$ при $n>0$ содержат соответственно обе функции Бесселя $I_{\nu+s}, K_{\nu+s}$ и $J_{\nu+s}, Y_{\nu+s}$.

Исследуем поведение компонент $f_{\lambda n}^{ \pm}(v)$ и $\theta_{\lambda n}^{ \pm}(v)$ в двух пределах: $v \rightarrow 0$ и $v \rightarrow \infty$. Для этого используем представления (44)-(46), асимптотики функций Бесселя [9] и функцию

$$
\vartheta_{\mu}(v) \equiv v-\frac{\pi}{2}\left(\mu+\frac{1}{2}\right)
$$

Если $2 \lambda \geqslant-1$ и $n \geqslant 0$, то компонента $f_{\lambda n}^{ \pm}, n \geqslant 0$, удовлетворяет соотношениям

$$
f_{\lambda n}^{ \pm}(v) \sim v^{2 n+\nu}, \quad v \rightarrow 0 ; \quad f_{\lambda n}^{ \pm}(v) \sim v^{3 n-1 / 2}\left\{\begin{array}{c}
e^{v} \\
\sin \vartheta_{\nu+3 n}(v)
\end{array}\right\}, \quad \frac{v}{\nu+3 n} \rightarrow \infty
$$

Асимптотическое поведение компоненты $\theta_{\lambda n}^{ \pm}$радикально зависит от индексов $\lambda$ и $n$. И для $2 \lambda>-1$, и для $2 \lambda=-1$ при любом $n \geqslant 1$ верны формулы

$$
\theta_{\lambda n}^{ \pm}(v) \sim v^{-\nu}, \quad v \rightarrow 0 ; \quad \theta_{\lambda n}^{ \pm}(v) \sim v^{3 n-1 / 2}\left\{\begin{array}{c}
e^{v} \\
\cos \vartheta_{\nu+3 n}(v)
\end{array}\right\}, \quad \frac{v}{\nu+3 n} \rightarrow \infty .
$$

Для $2 \lambda>-1$ и $n=0$ первая из этих формул остается в силе, а вторая вместо растущей экспоненты $e^{v}$ содержит убывающую экспоненту $e^{-v}$ :

$$
\theta_{\lambda 0}^{ \pm}(v) \sim v^{-\nu}, \quad v \rightarrow 0 ; \quad \theta_{\lambda 0}^{ \pm}(v) \sim v^{-1 / 2}\left\{\begin{array}{c}
e^{-v} \\
\cos \vartheta_{\nu}(v)
\end{array}\right\}, \quad \frac{v}{\nu} \rightarrow \infty
$$


В оставшемся случае $2 \lambda=-1$ и $n=0$ выполняются соотношения

$$
\theta_{\lambda 0}^{ \pm}(v) \sim \ln v, \quad v \rightarrow 0 ; \quad \theta_{\lambda 0}^{ \pm}(v) \sim v^{-1 / 2}\left\{\begin{array}{c}
e^{-v} \\
\cos \vartheta_{0}(v)
\end{array}\right\}, \quad v \rightarrow \infty
$$

Обсудим асимптотики (47)-(50). Если $2 \lambda>-1$ и $n>0$, то компоненты $f_{\lambda n}^{+}$и $f_{\lambda n}^{-}$ при $v \rightarrow 0$ сходятся к нулю по одному и тому же закону $f_{\lambda n}^{ \pm} \sim v^{\nu+2 n}$, а модули компонент $\theta_{\lambda n}^{+}$и $\theta_{\lambda n}^{-}$возрастают одинаковым образом: $\left|\theta_{\lambda n}^{ \pm}\right| \sim v^{-\nu}$. В случае $2 \lambda>-1$ и $n>0$ компоненты $f_{\lambda n}^{+}, \theta_{\lambda n}^{+}$и компоненты $f_{\lambda n}^{-}, \theta_{\lambda n}^{-}$имеют радикально разные асимптотики при $v \rightarrow 0$. Действительно, модули компонент $f_{\lambda n}^{+}$и $\theta_{\lambda n}^{+}$быстро растут как произведение функций $v^{3 n-1 / 2}$ и $e^{v}$, а компоненты $f_{\lambda n}^{-}$и $\theta_{\lambda n}^{-}$осциллируют с амплитудой порядка $O\left(v^{3 n-1 / 2}\right)$. В случае $2 \lambda=-1, n=0$ компоненты $\theta_{\lambda 0}^{+}$и $\theta_{\lambda 0}^{-}$ расходятся при $v \rightarrow 0$, как $\ln v$.

Теперь решим следующую задачу: в случае $2 \lambda>-1, \ell+p=n>0, p>0$ и $v \geqslant 0$ найти ограничения на потенциал $V$, достаточные для сходимости интегралов

$$
\gamma^{+}(v) \equiv \int_{0}^{v} t^{3} V(t) f_{\lambda \ell}^{+}(t) \theta_{\lambda p}^{+}(t) d t, \quad \gamma^{-}(v) \equiv \int_{0}^{v} t^{3} V(t) f_{\lambda \ell}^{-}(t) \theta_{\lambda p}^{-}(t) d t .
$$

Решение начнем с пояснения: интеграл $\gamma^{+}$отвечает случаю кулоновского отталкивания $(\alpha=1)$, а интеграл $\gamma_{1 n}^{-}-$случаю кулоновского притяжения $(\alpha=-1)$.

Пусть $v \rightarrow 0$, тогда $f_{\lambda \ell}^{ \pm} \theta_{\lambda p}^{ \pm} \sim O\left(v^{2 \ell}\right)$. Поэтому одни и те же условия $(1)$, не зависящие от $\alpha$, являются достаточными для сходимости обоих интегралов (51) на нижнем пределе и на интервале $0<v<\infty$. Теперь пусть $v \rightarrow \infty$, тогда

$$
t^{3}|V(t)|\left|f_{\lambda \ell}^{+}(t) \theta_{\lambda p}^{+}(t)\right| \sim t^{3 n+2}|V(t)| e^{2 t}, \quad t^{3}|V(t)|\left|f_{\lambda \ell}^{-}(t) \theta_{\lambda p}^{-}(t)\right| \sim t^{3 n+2}|V(t)| .
$$

Поэтому условия, достаточные для сходимости интегралов $\gamma^{+}$и $\gamma^{-}$при $v=\infty$, радикально разные: интеграл $\gamma^{+}$заведомо сходится при условиях $(2)$ с $\alpha=1$, $2 \beta>3(n+1)$, а интеграл $\gamma^{-}-$при более слабых условиях $(2)$ с $\alpha=-1,2 \beta>3(n+1)$. Следовательно, зависимость условий (2) от параметра $\alpha$ порождается тем, что в пределе $v \rightarrow \infty$ поведение компонент $f_{\lambda k}^{+}$и $\theta_{\lambda k}^{+}$отличается от поведения компонент $f_{\lambda k}^{-}$ и $\theta_{\lambda k}^{-}$. При условиях $(2)$ с $\alpha= \pm 1$, но при $2 \beta \leqslant 3(n+1)$ оба интеграла (51) могут расходиться в точке $v=\infty$.

4.2. Леммы о мажорантных оценках. Рассмотрим два случая: $2 \lambda>-1$ и $2 \lambda=-1$. В каждом из них докажем лемму о мажорантной оценке произведения $p_{1}^{ \pm} p_{2}^{ \pm}$функций $(38)$.

ЛЕмма 1. Пусть $2 \lambda>-1$ и по определению

$$
\ell(v) \equiv a_{\lambda} \int_{0}^{v} t^{3}|V(t)| d t, \quad a_{\lambda} \equiv \sqrt{\frac{2 \pi}{2 \lambda+1}}, \quad 2 \tau(v) \equiv e^{2 \ell(v)}-1 .
$$

Тогда при условиях (8) произведение $p_{1}^{ \pm} p_{2}^{ \pm}$функиий (38) удовлетворяет неравенству

$$
\left|p_{1}^{ \pm}(v) p_{2}^{ \pm}(w)\right| \leqslant \partial_{v} \tau(v) \partial_{w} \tau(w), \quad 0 \leqslant w \leqslant v \leqslant \infty .
$$


ДокАЗАТЕЛЬство начнем с анализа интеграла $\ell$ и функции $\tau$. Вследствие условий (8) интеграл $\ell$ сходится на нижнем пределе, существует при любом $v>0$, в том числе и при $v=\infty$. Поэтому из определения (52) функции $\tau$ через интеграл $\ell$ вытекают следующие ее важные свойства: она равна нулю в точке $v=0$, монотонно возрастает, но ограничена на полуоси $v \geqslant 0$; производная $\partial_{v} \tau$ ограничена при $v>0$, вообще говоря, не ограничена в точке $v=0$, но интегрируема на всей полуоси $v \geqslant 0$.

Продолжим доказательство. В известной мажорантной оценке [5]

$$
\left|F_{\lambda}\left(\rho^{\prime}, \eta\right) G_{\lambda}(\rho, \eta)\right| \leqslant a_{\lambda} \sqrt{\rho^{\prime} \rho}, \quad 0 \leqslant \rho^{\prime} \leqslant \rho \leqslant \infty, \quad 2 \lambda>-1,
$$

заменим функцию $G_{\lambda}$ суммой $(19)$, функции $F_{\lambda}$ и $\Theta_{\lambda}$ выразим через функции $f_{\lambda}^{ \pm}$ и $\theta_{\lambda}^{ \pm}$по формулам $(21)$, воспользуемся свойствами $(20),(23)$ и $(24)$ функций $h^{\text {с }}$ и $P_{\lambda}$ и, наконец, представим функции $f_{\lambda}^{ \pm}$и $\theta_{\lambda}^{ \pm}$рядами (33). В полученном неравенстве положим $\rho=v^{2} / 8|\eta|, \rho^{\prime}=w^{2} / 8|\eta|$ и перейдем к пределу $q \rightarrow 0$. В итоге получим оценку

$$
\left|f_{\lambda 0}^{ \pm}(w) \theta_{\lambda 0}^{ \pm}(v)\right| \leqslant a_{\lambda}, \quad 0 \leqslant w \leqslant v \leqslant \infty, \quad 2 \lambda>-1 .
$$

Перейдем к выводу других вспомогательных соотношений. Благодаря оценке (54) интеграл $b^{ \pm}$, заданный формулой $(37)$, удовлетворяет следующей цепочке неравенств:

$$
\begin{aligned}
\left|b^{ \pm}(v)\right|=\left|\int_{0}^{v} t^{3} V(t) f_{\lambda 0}^{ \pm}(t) \theta_{\lambda 0}^{ \pm}(t) d t\right| & \leqslant \int_{0}^{v} t^{3}|V(t)|\left|f_{\lambda 0}^{ \pm}(t) \theta_{\lambda 0}^{ \pm}(t)\right| d t \leqslant \\
& \leqslant a_{\lambda} \int_{0}^{v} t^{3}|V(t)| d t=\ell(v)<\infty, \quad v \geqslant 0 .
\end{aligned}
$$

Следовательно, $\left|b^{ \pm}(v)\right| \leqslant \ell(v)$ при $v \geqslant 0$, и поэтому при $v \geqslant 0$ верны неравенства

$$
e^{-2 b^{ \pm}(v)} \leqslant e^{2 \ell(v)}<\infty, \quad e^{2 b^{ \pm}(v)} \leqslant e^{2 \ell(v)}<\infty .
$$

Вследствие этих неравенств и определений (52) при любом $v \geqslant 0$ имеем

$$
a_{\lambda} v^{3}|V(v)| e^{ \pm 2 b^{ \pm}(v)} \leqslant a_{\lambda} v^{3}|V(v)| e^{2 \ell(v)}=\partial_{v} \ell(v) e^{2 \ell(v)}=\partial_{v} \tau(v) .
$$

Теперь докажем неравенство (53). Используя определение (38) функций $p_{1}^{ \pm}$и $p_{2}^{ \pm}$, оценку (54) и соотношения (55), при условии $0 \leqslant w \leqslant v \leqslant \infty$ получаем

$$
\begin{aligned}
\left|p_{1}^{ \pm}(v) p_{2}^{ \pm}(w)\right| & \leqslant\left\{\left|v^{3} V(v)\right| e^{ \pm 2 b^{ \pm}(v)}\right\}\left\{w^{3}|V(w)| e^{-2 b^{ \pm}(w)}\right\}\left|\theta_{\lambda 0}^{ \pm}(v) f_{\lambda 0}^{ \pm}(w)\right|^{2} \leqslant \\
& \leqslant\left\{a_{\lambda} v^{3}|V(v)| e^{2 \ell(v)}\right\}\left\{a_{\lambda} w^{3}|V(w)| e^{2 \ell(w)}\right\}=\partial_{v} \tau(v) \partial_{w} \tau(w)
\end{aligned}
$$

что и требовалось показать.

Лемма 2. Пусть $2 \lambda=-1, \gamma-$ постоянная Эйлера, интеграл $\ell^{ \pm}$задан формулами

$$
\ell^{ \pm}(v)=\frac{1}{2} \int_{0}^{v} t^{3}|V(t)| \phi^{2}(t)\left\{\begin{array}{c}
\operatorname{ch}^{2} v \\
1
\end{array}\right\} d t, \quad \phi(v) \equiv\left|\ln \frac{t}{2}+\gamma\right|+1,
$$

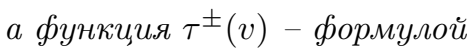

$$
2 \tau^{ \pm}(v)=e^{2 \ell^{ \pm}(v)}-1
$$

Тогда при условиях (1) и (2) с $\beta>2$ верна ощенка

$$
\left|p_{1}^{ \pm}(v) p_{2}^{ \pm}(w)\right| \leqslant \partial_{v} \tau^{ \pm}(v) \partial_{w} \tau^{ \pm}(w), \quad v \geqslant 0, \quad w \geqslant 0 .
$$


ДокАЗАТЕЛЬство начнем следующим замечанием: интеграл $\ell^{ \pm}$и функция $\tau^{ \pm}$ обладают теми же свойствами, что и интеграл $\ell$ и функция $\tau$, заданные формулой (52). При условиях (1) и (2) с $\beta>2$ интеграл $\ell^{ \pm}$сходится на нижнем пределе и существует при любом $v>0$, в том числе и при $v=\infty$. Согласно определению (57) функция $\tau^{ \pm}$равна нулю в точке $v=0$, монотонно возрастает, но ограничена на полуоси $v \geqslant 0$. Производная $\partial_{v} \tau^{ \pm}$ограничена в области $v>0$, вообще говоря, не ограничена в точке $v=0$, но интегрируема на всей полуоси $v \geqslant 0$.

Выведем заведомо завышенные, но зато удобные для дальнейших исследований мажорантные оценки функций Бесселя нулевого порядка. Как известно [9], в пределе $v \rightarrow \infty$ функции $Y_{0}$ и $K_{0}$ сходятся к нулю, а в пределе $v \rightarrow 0$ имеют однотипные асимптотики:

$$
Y_{0}(v) \sim \frac{2}{\pi}\left(\ln \frac{v}{2}+\gamma\right), \quad K_{0}(v) \sim-\left(\ln \frac{v}{2}+\gamma\right) .
$$

Поэтому можно предположить, что эти функции удовлетворяют соотношениям

$$
\left\{\begin{array}{c}
K_{0}(v) \\
\left|Y_{0}(v)\right|
\end{array}\right\} \leqslant \phi(v)\left\{\begin{array}{c}
\operatorname{ch} v \\
1
\end{array}\right\}, \quad v \geqslant 0
$$

В результате вычислений нам удалось показать, что эти соотношения справедливы. Известно [9], что $\left|J_{0}(v)\right| \leqslant 1$ и $I_{0}(v) \leqslant \operatorname{ch} v$ при $v \geqslant 0$. Следовательно, при $v \geqslant 0$

$$
\left\{\begin{array}{c}
I_{0}(v) \\
\left|J_{0}(v)\right|
\end{array}\right\} \leqslant \phi(v)\left\{\begin{array}{c}
\operatorname{ch} v \\
1
\end{array}\right\}
$$

Оценим модуль произведения $f_{\lambda 0}^{ \pm} \theta_{\lambda 0}^{ \pm}$сверху. Благодаря равенствам (46) и полученным выше оценкам функций Бесселя нулевого порядка имеет место равномерная и симметричная относительно перестановки аргументов $v$ и $w$ мажорантная оценка

$$
\left|f_{\lambda 0}^{ \pm}(v) \theta_{\lambda 0}^{ \pm}(w)\right| \leqslant \frac{1}{2} \phi(v) \phi(w)\left\{\begin{array}{c}
\operatorname{ch} v \operatorname{ch} w \\
1
\end{array}\right\}, \quad v \geqslant 0, \quad w \geqslant 0, \quad 2 \lambda=-1
$$

Теперь можно получить неравенства (58) по аналогии с неравенствами (53), вывод которых подробно изложен в лемме 1. Для этого вместо оценки (54) используем оценку (59), а вместо функций $\ell$ и $\tau-$ функции $\ell^{ \pm}$и $\tau^{ \pm}$. Ограничимся краткими пояснениями. В силу определений (37) и (56) интегралов $b^{ \pm}$и $\ell^{ \pm}$и оценки (59) при $v \geqslant 0$ верны три неравенства:

$$
\left|b^{ \pm}(v)\right| \leqslant \ell^{ \pm}(v), \quad e^{-2 b^{ \pm}(v)} \leqslant e^{2 \ell^{ \pm}(v)}, \quad e^{2 b^{ \pm}(v)} \leqslant e^{2 \ell^{ \pm}(v)} .
$$

Вследствие этих неравенств, определений (38), (57) функций $p_{1}^{ \pm}, p_{2}^{ \pm}, \tau^{ \pm}$и оценки (59) верно неравенство (58). Ключевыми при его выводе являются тождества

$$
2 \partial_{v} \tau^{ \pm}(v) \equiv 2 \partial_{v} \ell^{ \pm}(v) e^{2 \ell^{ \pm}(v)} \equiv v^{3}|V(v)| \phi^{2}(v)\left\{\begin{array}{c}
\operatorname{ch}^{2}(v) \\
1
\end{array}\right\} e^{2 \ell^{ \pm}(v)}
$$

Этим замечанием закончим доказательство леммы. 
4.3. Теоремы существования и единственности. Последовательно докажем теоремы существования и единственности непрерывного на полуоси решения $\left\{y_{1 n}^{ \pm}, y_{2 n}^{ \pm}\right\}$каждой (для $n=0,1, \ldots, m$ ) системы интегральных уравнений (43). Используя эти теоремы, покажем, что при $n=1,2, \ldots, m$ задача Коши (10), (11) имеет непрерывное на полуоси решение $\left\{c_{n}^{ \pm}, s_{n}^{ \pm}\right\}$. В случае $2 \lambda>-1, n=0$ существование и единственность решения системы системы интегральных уравнений (43) докажем подробно. Это позволит существенно сократить анализ случаев $2 \lambda>-1, n>0$ и $2 \lambda=-1, n \geqslant 0$.

Теорема 3. Если $2 \lambda>-1$ u $n=0$, то при условиях (8) система уравнений (43) имеет единственное решение $\left\{y_{10}^{ \pm}, y_{20}^{ \pm}\right\}$. Его компоненты $y_{10}^{ \pm}$и $y_{20}^{ \pm}$непрерывны на полуоси $v \geqslant 0$ и в пределе $v \rightarrow 0$ ведут себя, как $y_{10}^{ \pm} \rightarrow 1, y_{20}^{ \pm}=o\left(v^{2 \nu}\right), \nu=2 \lambda+1$.

ДокАЗАТЕЛЬСТВо начнем пояснениями. При $n=0$ из формул (40)-(43) следуют тождества $a_{i 0}^{ \pm}(v) \equiv 0, d_{i 0}^{ \pm}(v) \equiv \delta_{1, i}$ и $r_{i 0}(v) \equiv \delta_{1, i}$, где $i=1,2$, а $v \geqslant 0$. Поэтому первое (при $i=1, j=2$ ) уравнение системы (43) записывается как

$$
y_{10}^{ \pm}(v)=1+\int_{0}^{v} p_{1}^{ \pm}\left(v_{1}\right) d v_{1} \int_{0}^{v_{1}} p_{2}^{ \pm}\left(v_{2}\right) y_{10}^{ \pm}\left(v_{2}\right) d v_{2}, \quad v \geqslant 0
$$

второе (при $i=2, j=1$ ) уравнение системы (42) сводится к интегральному соотношению

$$
y_{20}^{ \pm}(v)=\int_{0}^{v} p_{2}^{ \pm}(t) y_{10}^{ \pm}(t) d t, \quad v \geqslant 0
$$

в результате первое уравнение системы принимает вид

$$
y_{10}^{ \pm}(v)=1+\int_{0}^{v} p_{1}^{ \pm}(t) y_{20}^{ \pm}(t) d t, \quad v \geqslant 0 .
$$

Исследуем уравнение (60) методом последовательных приближений Пикара-Линделёфа [8]. Определим элементы $z_{m}^{ \pm}(v)$ бесконечной итерационной последовательности $\left\{z_{m}^{ \pm}\right\}_{m=0}^{\infty}$. Пусть $z_{0}^{ \pm}(v) \equiv 1$ при любом $v \geqslant 0$, а каждый элемент $z_{m}^{ \pm}$(для $m=1,2, \ldots)$ есть правая часть уравнения $(60)$, в которой искомая компонента $y_{1}^{ \pm}$ заменена элементом $z_{m-1}^{ \pm}$:

$$
z_{m}^{ \pm}(v)=1+\int_{0}^{v} p_{1}^{ \pm}\left(v_{1}\right) d v_{1} \int_{0}^{v_{1}} p_{2}^{ \pm}\left(v_{2}\right) z_{m-1}^{ \pm}\left(v_{2}\right) d v_{2}, \quad m \geqslant 1 .
$$

Следовательно, элемент $z_{m}^{ \pm}$является частичной суммой $W_{m}^{ \pm}$слагаемых $z_{0}^{ \pm} \equiv 1$ и $w_{k}^{ \pm}$ с номерами $k=1, \ldots, m$, где

$$
w_{k}^{ \pm}(v) \equiv \int_{0}^{v} p_{1}\left(v_{1}\right) d v_{1} \int_{0}^{v_{1}} p_{2}^{ \pm}\left(v_{2}\right) d v_{2} \cdots \int_{0}^{v_{2 k-2}} p_{1}^{ \pm}\left(v_{2 k-1}\right) d v_{2 k-1} \int_{0}^{v_{2 k-1}} p_{2}^{ \pm}\left(v_{2 k}\right) d v_{2 k}
$$

бесконечного ряда

$$
W^{ \pm}(v)=1+\sum_{k=1}^{\infty} w_{k}^{ \pm}(v) .
$$

Поэтому итерационная последовательность $\left\{z_{m}^{ \pm}\right\}_{m=0}^{\infty}$ имеет предел $z_{\infty}^{ \pm}=W^{ \pm}$, являющийся искомым решением $y_{1}^{ \pm}$, тогда и только тогда, когда сходится ряд $W^{ \pm}$. 
Покажем, что ряд $W^{ \pm}$сходится на всей полуоси $v \geqslant 0$, причем абсолютно и равномерно. Начнем с оценки модуля каждого слагаемого $w_{k}^{ \pm}, k \geqslant 1$, этого ряда. По определению (63) слагаемое $w_{k}^{ \pm}$есть интеграл кратности $2 k$. Он содержит $k$ произведений функций $p_{1}^{ \pm}\left(v_{p}\right)$ и $p_{2}^{ \pm}\left(v_{p+1}\right), p=1,2, \ldots, 2 k-1$, с аргументами $v_{p}$ и $v_{p+1}$, удовлетворяющими неравенству $v_{p+1} \leqslant v_{p}$. Согласно определениям (52) и неравенству (53) модуль такого произведения не превосходит произведения производных $\partial_{v_{p}} \tau\left(v_{p}\right)$ и $\partial_{v_{p+1}} \tau\left(v_{p+1}\right)$, а функция $\tau(v)$ равна нулю в точке $v=0$. Поэтому из равенства (63) следует равномерная по переменной $v$ и довольно простая мажорантная оценка

$$
\begin{aligned}
\left|w_{k}^{ \pm}(v)\right| \leqslant \int_{0}^{v} \partial_{v_{1}} \tau\left(v_{1}\right) d v_{1} \int_{0}^{v_{1}} \partial_{v_{2}} \tau\left(v_{2}\right) d v_{2} \cdots & \\
& \ldots \int_{0}^{v_{2 k-2}} \partial_{v_{2 k-1}} \tau\left(v_{2 k-1}\right) d v_{2 k-1} \int_{0}^{v_{2 k-1}} \partial_{v_{2 k}} \tau\left(v_{2 k}\right) d v_{2 k}=\frac{\tau^{2 k}(v)}{(2 k) !}
\end{aligned}
$$

Используя ее и известное разложение гиперболического косинуса $\operatorname{ch} \tau$, получим следующую равномерную по аргументу $v$ оценку ряда (64):

$$
\left|W^{ \pm}(v)\right| \leqslant 1+\sum_{k=1}^{\infty}\left|w_{k}(v)\right| \leqslant 1+\sum_{k=1}^{\infty} \frac{\tau^{2 k}(v)}{(2 k) !}=\operatorname{ch} \tau(v)<\infty, \quad v \geqslant 0 .
$$

Следовательно, функция $\operatorname{ch} \tau$, аргумент которой $\tau$ принимает только конечные значения, есть ограниченная мажоранта ряда $W^{ \pm}$. Поэтому на полуоси $v \geqslant 0$ ряд $W^{ \pm}$ сходится равномерно и абсолютно по мажорантному признаку Вейерштрасса [10].

Продолжим исследование ряда $W^{ \pm}$. Оценка (65) означает, что при любом значении $k=1,2, \ldots$ интеграл в правой части равенства (63) сходится для всех $v \geqslant 0$. Следовательно, левая часть этого равенства - функция $w_{k}^{ \pm}-$непрерывна при любых $k=1,2, \ldots$ на полуоси $v \geqslant 0$.

Итак, при $v \geqslant 0$ ряд $W^{ \pm}$сходится абсолютно и равномерно, а все его слагаемые $w_{k}^{ \pm}$- непрерывные функции. Согласно теории рядов [10] он является непрерывной функцией на полуоси $v \geqslant 0$. В силу определений (63) и (64) слагаемых $w_{n}^{ \pm}$ и их суммы $W^{ \pm}$эта сумма является решением $y_{10}^{ \pm}=W^{ \pm}$уравнения (60).

Покажем от противного, что это уравнение не имеет другого решения, непрерывного при $v \geqslant 0$. Пусть $\tilde{y}_{10}^{ \pm}$- еще одно решение. Тогда разность $\zeta^{ \pm}=y_{10}^{ \pm}-\tilde{y}_{10}^{ \pm}$ удовлетворяет тождеству

$$
\zeta^{ \pm}(v) \equiv \int_{0}^{v} p_{1}^{ \pm}\left(v_{1}\right) d v_{1} \int_{0}^{v_{1}} p_{2}^{ \pm}\left(v_{2}\right) \zeta^{ \pm}\left(v_{2}\right) d v_{2}, \quad v \geqslant 0 .
$$

После $k \geqslant 2$ итераций это тождество благодаря равенству (63) станет тождеством

$$
\zeta^{ \pm}(v) \equiv \int_{0}^{v} p_{1}^{ \pm}\left(v_{1}\right) d v_{1} \int_{0}^{v_{1}} p_{2}^{ \pm}\left(v_{2}\right) w_{k-1}^{ \pm}\left(v_{2}\right) \zeta^{ \pm}\left(v_{2}\right) d v_{2}, \quad k=2,3, \ldots, \quad v \geqslant 0 .
$$

Данное тождество и оценки $(53),(65)$ функций $p_{1}^{ \pm} p_{2}^{ \pm}$и $w_{k-1}^{ \pm}$порождают неравенство

$$
\left|\zeta^{ \pm}(v)\right| \leqslant \max _{0 \leqslant t<v}\left|\zeta^{ \pm}(t)\right| \frac{\tau^{2 k}(v)}{(2 k) !}, \quad k=2,3, \ldots, \quad v \geqslant 0 .
$$


В пределе $k \rightarrow \infty$ оно вырождается в соотношение $\left|\zeta^{ \pm}(v)\right| \leqslant 0$ при $v \geqslant 0$, из которого следует, что $y_{10}^{ \pm}=\tilde{y}_{10}^{ \pm}$при любом $v \geqslant 0$. Поэтому исследуемое уравнение $(60)$ имеет только одно решение $y_{10}^{ \pm}=W^{ \pm}$.

Теперь построим и исследуем решение $y_{20}^{ \pm}$второго уравнения системы (43). Это решение является интегралом (61). Положив в этом интеграле $y_{10}^{ \pm}=W^{ \pm}$, получаем интегральное представление искомого решения $y_{20}^{ \pm}$через уже найденный ряд $W^{ \pm}$:

$$
y_{20}^{ \pm}(v)=\int_{0}^{v} p_{2}^{ \pm}(t) W^{ \pm}(t) d t, \quad v \geqslant 0 .
$$

Обсудим подынтегральную функцию в (66). На всей полуоси $v \geqslant 0$ ряд $W^{ \pm}$есть ограниченная и непрерывная функция. В определении (38) потенциал $V$ удовлетворяет условиям (8), функции $f_{\lambda 0}^{+}$или $f_{\lambda 0}^{-}$непрерывны на интервале $0 \leqslant v<\infty$ и имеют асимптотики (47), а интеграл $b^{ \pm}$непрерывен на полуоси $v \geqslant 0$. Поэтому при любом $v \geqslant 0$ интеграл (66) сходится и является непрерывной функцией.

Перейдем к выводу соотношения $y_{20}^{ \pm}(v)=o\left(v^{4 \lambda+2}\right)$ при $v \rightarrow 0$. Так как функция $y_{10}^{ \pm}$в точке $v=0$ непрерывна справа и равна единице, $y_{10}^{ \pm}(v) \sim 1+o(1)$ при $v \rightarrow 0$. Покажем, что в этом пределе функция $y_{20}^{ \pm}$быстро сходится к нулю. Для этого в представлении (61) положим $y_{10}^{ \pm}(v)=1+o(1)$, воспользуемся определением $(38)$ функции $p_{2}^{ \pm}$и заменим функцию $f_{\lambda 0}^{ \pm}$ее асимптотикой $(47)$ при $v \rightarrow 0$. Таким образом получим старшее слагаемое асимптотики функции $y_{20}^{ \pm}$в виде интеграла:

$$
y_{20}^{ \pm}(v) \sim-\int_{0}^{v} t^{4 \lambda+5} V(t) d t, \quad v \rightarrow 0 .
$$

Такой интеграл убывает, как $o\left(v^{4 \lambda+2}\right)$. Действительно, по правилу Лопиталя [10]

$$
\lim _{v \rightarrow 0} v^{-4 \lambda-2} \int_{0}^{v} t^{4 \lambda+5} V(t) d t=\frac{1}{4 \lambda+2} \lim _{v \rightarrow 0} v^{4} V(v)=0 .
$$

Осталось убедиться в том, что интеграл (66) - единственное решение второго уравнения системы (43) в классе функций, непрерывных на полуоси $v \geqslant 0$. Предположим противное: пусть имеется еще одно решение $\tilde{y}_{20}^{ \pm}$. Тогда согласно равенству (62) функция $y_{10}^{ \pm}=W^{ \pm}$является интегральным образом как функции $y_{20}^{ \pm}$, так и функции $\tilde{y}_{20}^{ \pm}$:

$$
W^{ \pm}(v)=1+\int_{0}^{v} p_{1}^{ \pm}(t) y_{20}^{ \pm}(t) d t=1+\int_{0}^{v} p_{1}^{ \pm}(t) \tilde{y}_{20}^{ \pm}(t) d t, \quad v \geqslant 0 .
$$

Следовательно, разность $y_{20}^{ \pm}-\tilde{y}_{20}^{ \pm}$удовлетворяет тождеству

$$
\int_{0}^{v} p_{1}^{ \pm}(t)\left[y_{20}^{ \pm}(t)-\tilde{y}_{20}^{ \pm}(t)\right] d t \equiv 0, \quad v \geqslant 0 .
$$

После дифференцирования по аргументу $v$ это тождество становится соотношением

$$
p_{1}^{ \pm}(v)\left[y_{20}^{ \pm}(v)-\tilde{y}_{20}^{ \pm}(v)\right] \equiv 0, \quad v \geqslant 0,
$$

которое выполняется только в случае $y_{20}^{ \pm}(v) \equiv \tilde{y}_{20}^{ \pm}(v), v \geqslant 0$. Следовательно, интеграл (66) - единственное непрерывное при $v \geqslant 0$ решение второго уравнения системы (43). Это утверждение завершает доказательство теоремы и анализ системы (43) при $2 \lambda>-1$ и $n=0$. 
Перейдем к исследованию системы (43) в случае $2 \lambda>-1, n>0$. В этом случае согласно равенствам (40)-(43) слагаемые $r_{1 n}^{ \pm}$и $r_{2 n}^{ \pm}$являются довольно сложными функциями. Поэтому сначала необходимо доказать лемму о предполагаемых свойствах таких слагаемых.

ЛЕмма 3. Предположим, что в случае $2 \lambda>-1, n>0$ все функиии $y_{1 k}^{ \pm}$и $y_{2 k}^{ \pm}$ в суммах (41) непрерывны при $v \geqslant 0$ и имеют асимптотики $y_{1 k}^{ \pm}=o(1), y_{2 k}=o\left(v^{2 \nu}\right)$ при $v \rightarrow 0$. Тогда при условиях (1) u (2) с $2 \beta>3(n+1)$ слагаемые $r_{1 n}^{ \pm}$u $r_{2 n}^{ \pm}$ системы (43) непрерьвны на полуоси $v \geqslant 0$ и имеют асимптотики $r_{1 n}^{ \pm}=o(1)$ $u r_{2 n}^{ \pm}=o\left(v^{2 \nu}\right)$ npu $v \rightarrow 0$.

ДокАЗАтЕЛЬство. Нам потребуются асимптотики (47)-(49) компонент $f_{\lambda n}^{ \pm}$и $\theta_{\lambda n}^{ \pm}$, определения (41)-(43) функций $a_{i n}^{ \pm}, d_{i n}^{ \pm}, r_{i n}^{ \pm}$, представления (38) функций $p_{1}^{ \pm}$и $p_{2}^{ \pm}$, а также свойства функций $y_{10}^{ \pm}$и $y_{20}^{ \pm}$, установленные в теореме 3 . Исследуем функции $a_{1 n}^{ \pm}, a_{2 n}^{ \pm}$и интегралы $d_{1 n}^{ \pm}, d_{2 n}^{ \pm}$по аналогии с приведенным в п. 4.1 анализом интегралов (51).

Пусть $v \rightarrow 0$. Тогда при условиях (1) и любых $k$ и $\ell \leqslant n-1$ имеем

$v^{3} V(v)=o\left(v^{-1}\right), \quad f_{\lambda \ell}^{ \pm}=o\left(v^{2 \nu+2 \ell}\right), \quad \theta_{\lambda \ell}^{ \pm}=o\left(v^{-2 \nu}\right), \quad y_{1 k}^{ \pm}=\delta_{0, k}+o(1), \quad y_{2 k}^{ \pm}=o\left(v^{2 \nu}\right)$.

Поэтому суммы $a_{1 n}^{ \pm}$и $a_{2 n}^{ \pm}$подчиняются следующим соотношениям:

$a_{1 n}^{ \pm} \sim y_{10}^{ \pm} v^{3} V f_{\lambda 0}^{ \pm} \theta_{\lambda n}^{ \pm}=o\left(v^{-1}\right), \quad a_{2 n}^{ \pm} \sim v^{3} V f_{\lambda 0}^{ \pm} \sum_{k=0}^{n-1} y_{2 k}^{ \pm} \sum_{p=n-k} \theta_{\lambda p}^{ \pm}=o\left(v^{2 \nu-1}\right), \quad v \rightarrow 0$.

Следовательно, при $v \rightarrow 0$ интегралы $d_{1 n}^{ \pm}$и $d_{2 n}^{ \pm}$от сумм $a_{1 n}^{ \pm}$и $a_{2 n}^{ \pm}$имеют при $v \rightarrow 0$ асимптотики $d_{1 n}^{ \pm}=o(1), d_{2 n}^{ \pm}=o\left(v^{2 \nu}\right)$, а функции $p_{1}^{ \pm}$и $p_{2}^{ \pm}-$асимптотики $p_{1}^{ \pm}=$ $o\left(v^{-2 \nu-1}\right)$ и $p_{2}^{ \pm}=o\left(v^{2 \nu-1}\right)$. Слагаемые $r_{1 n}^{ \pm}$и $r_{2 n}^{ \pm}$содержат только интегралы $d_{1 n}^{ \pm}, d_{1 n}^{ \pm}$ и функции $p_{1}^{ \pm}, p_{2}^{ \pm}$, поэтому $r_{1 n}^{ \pm}=o(1)$, а $r_{2 n}^{ \pm}=o\left(v^{2 \nu}\right)$ при $v \rightarrow 0$.

Итак, при условиях (1) функции $d_{i n}^{ \pm}$и $r_{i n}^{ \pm}, i=1,2$, сходятся к нулю в точке $v=0$ и являются непрерывными на интервале $0 \leqslant v<\infty$. Осталось исследовать поведение этих функций в пределе $v \rightarrow \infty$.

Пусть $v \rightarrow \infty$. Тогда в суммах $a_{1 n}^{ \pm}$и $a_{2 n}^{ \pm}$все функции $y_{1 k}^{ \pm}$и $y_{2 k}^{ \pm}, k \leqslant n-1$, сходятся к своим конечным значениям в точке $v=\infty$, а асимптотики всех произведений $f_{\lambda \ell}^{ \pm} f_{\lambda p}^{ \pm}, f_{\lambda \ell}^{ \pm} \theta_{\lambda p}^{ \pm}$и $\theta_{\lambda \ell}^{ \pm} \theta_{\lambda p}^{ \pm}$содержат в качестве множителя степенную функцию $v^{3(\ell+p)-1}$ с $\ell+p=n=k$. Ее показатель принимает максимальное значение, равное $3 n-1$, при $k=0$. Поэтому

$$
a_{i n}^{ \pm}(v) \sim v^{3 n+2} V(v)\left\{\begin{array}{c}
e^{2 v} \\
1
\end{array}\right\}, \quad i=1,2, \quad v \rightarrow \infty .
$$

Потенциал $V$ удовлетворяет условию $(2)$ с $2 \beta>3(n+1)$, поэтому в пределе $v \rightarrow \infty$ суммы $a_{1 n}^{ \pm}$и $a_{2 n}^{ \pm}$сходятся к нулю быстрее функции $v^{-1}$. В результате интегралы $d_{1 n}^{ \pm}$и $d_{2 n}^{ \pm}$от таких сумм в точке $v=\infty$ принимают конечные значения. Таким образом, функции $r_{1 n}^{ \pm}$и $r_{2 n}^{ \pm}$непрерывны в точке $v=\infty$ слева, если интегралы от функций $\left|p_{1}^{ \pm}(t)\right|$ и $\left|p_{2}^{ \pm}(t)\right|$ по области $v>0$ сходятся на верхнем пределе $v=\infty$. Для такой сходимости достаточными являются условия (1) и условие (2) с $\beta>2$. Действительно, благодаря таким условиям и асимптотикам $(47)$ и $(48)$ компонент $f_{\lambda 0}^{ \pm}$ 
и $\theta_{\lambda 0}^{ \pm}$функции $p_{1}^{ \pm}$и $p_{2}^{ \pm}$непрерывны в области $0<v<\infty$ и сходятся к нулю при $v \rightarrow \infty$ быстрее функции $v^{-1}$.

Сформулируем два вывода. Если $V$ удовлетворяет условию $(2)$ с $2 \beta>3(n+1)$, то все функции $a_{i n}^{ \pm}, d_{i n}^{ \pm}$и $r_{i n}^{ \pm}$ограничены в точке $v=\infty$. В случае $2 \beta \leqslant 3(n+1)$ интегралы $d_{1 n}^{ \pm}$и $d_{2 n}^{ \pm}$, а следовательно, и функции $r_{1 n}^{ \pm}$и $r_{2 n}^{ \pm}$, вообще говоря, не имеют определенных пределов в точке $v=\infty$. Этими выводами завершим доказательство леммы.

Теорема 4. Пусть $2 \lambda>-1, n=1,2, \ldots, m$, и пусть выполнены условия (1)

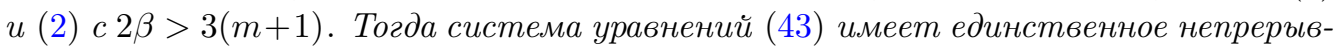
ное на полуоси $v \geqslant 0$ решение $\left\{y_{1 n}^{ \pm}, y_{2 n}^{ \pm}\right\}$, причем такое, что $y_{1 n}^{ \pm}=o(1), y_{2 n}^{ \pm}=o\left(v^{2 \nu}\right)$ $n p u v \rightarrow 0$.

ДоКАЗАТЕЛЬСТВо. Используем леммы 1 и 3 и применим метод последовательных приближений и метод математической индукции.

Пусть $n=1$. Методом последовательных приближений построим формальное решение $y_{1 n}^{ \pm}$первого (при $i=1, j=2$ ) уравнения системы (43) в виде бесконечного ряда

$$
X^{ \pm}(v)=r_{1 n}^{ \pm}(v)+\sum_{k=1}^{\infty} x_{k}^{ \pm}(v)
$$

со слагаемыми

$$
\begin{aligned}
x_{k}^{ \pm}(v) \equiv \int_{0}^{v} p_{1}^{ \pm}\left(v_{1}\right) d v_{1} \int_{0}^{v_{1}} p_{2}^{ \pm}\left(v_{2}\right) d v_{2} \ldots \\
\quad \ldots \int_{b}^{v_{2 k-2}} p_{1}^{ \pm}\left(v_{2 k-1}\right) d v_{2 k-1} \int_{0}^{v_{2 k-1}} p_{1}^{ \pm}\left(v_{2 k}\right) r_{1 n}^{ \pm}\left(v_{2 k}\right) d v_{2 k}, \quad k \geqslant 1 .
\end{aligned}
$$

Исследуем ряд $X^{ \pm}$. Согласно лемме 3 слагаемое $r_{1 n}^{ \pm}$с $n=1$ является всюду (на полуоси $v \geqslant 0$ ) непрерывной функцией. Максимальное значение $T_{0}^{ \pm}$модуля такой функции есть конечное число. Построим мажорантную оценку $T_{k}^{ \pm}$слагаемого $x_{k}^{ \pm}$ с номером $k \geqslant 1$. Сначала в интеграле (68) заменим все функции их модулями. Затем заменим функцию $\left|r_{1 n}^{ \pm}\right|$числом $T_{0}^{ \pm}$, а каждое (для $p=1,2, \ldots, 2 k-1$ ) произведение функций $\left|p_{1}^{ \pm}\left(v_{p}\right)\right|$ и $\left|p_{2}^{ \pm}\left(v_{p+1}\right)\right|$ заменим превосходящим его согласно неравенству (53) произведением производных $\partial_{v_{p}} \tau\left(v_{p}\right)$ и $\partial_{v_{p+1}} \tau\left(v_{p+1}\right)$. Используя равенство $\tau(v)=0$ при $v=0$, вычислим полученный интеграл $T_{k}^{ \pm}$и запишем искомую оценку в следующем виде:

$$
\left|x_{k}^{ \pm}(v)\right| \leqslant T_{k}^{ \pm}(v) \equiv T_{0}^{ \pm} \frac{\tau^{2 k}(v)}{(2 k) !}<\infty, \quad k=1,2, \ldots, \quad v \geqslant 0 .
$$

Благодаря такой оценке модуль $\left|X^{ \pm}\right|$ряда (67) не превосходит $T_{0}^{ \pm} \operatorname{ch} \tau$ на всей полуоси $v \geqslant 0$. Поэтому ряд $X^{ \pm}$сходится при $v \geqslant 0$ абсолютно и равномерно и является непрерывным решением $y_{1 n}^{ \pm}=X^{ \pm}$первого (при $i=1, j=2$ ) уравнения системы (43).

Исследуем поведение этого решения при $v \rightarrow 0$. В этом пределе согласно лемме 1 функция $\tau$ сходится к нулю, поэтому из оценки (69) следует, что все слагаемые $x_{k}^{ \pm}$ ряда $X^{ \pm}$убывают быстрее, чем $r_{1 n}^{ \pm}$. Согласно лемме 3 при $n=1$ верно соотношение $r_{1 n}^{ \pm}=o(1)$. Поэтому $X^{ \pm} \sim r_{1 n}^{ \pm}=o(1)$ и $y_{1 n}^{ \pm}=o(1)$ при $v \rightarrow 0$. 
Теперь построим решение $y_{2 n}^{ \pm}$второго (при $i=2, j=1$ ) уравнения системы (43) как образ (42) ряда (67):

$$
y_{2 n}^{ \pm}(v)=d_{2 n}^{ \pm}(v)+\int_{0}^{v} p_{2}^{ \pm}(t) X^{ \pm}(t) d t .
$$

Заменой $X \rightarrow d_{1 n}^{ \pm}$это равенство сводится к уже исследованной в лемме 3 функции $r_{2 n}^{ \pm}$(см. определение (43)). Обе функции $X^{ \pm}$и $d_{1 n}^{ \pm}$непрерывны на полуоси $v \geqslant 0$ и убывают, как $o(1)$ при $v \rightarrow 0$. Поэтому функции $r_{2 n}^{ \pm}$и $y_{2 n}^{ \pm}$обладают одинаковыми свойствами: они непрерывны на полуоси $v \geqslant 0$ и убывают, как $o\left(v^{2 \nu}\right)$ при $v \rightarrow 0$.

Итак, системе (43) с номером $n=1$ удовлетворяют ряд $X^{ \pm}$и его образ (70). Другого непрерывного на полуоси $v \geqslant 0$ решения не существует, что нетрудно показать от противного способами, подробно изложенными при доказательстве теоремы 3. Этим замечанием завершим доказательство теоремы в случае $n=1$.

Согласно теореме 3 и приведенному выше анализу случая $n=1$ компоненты $y_{1 n}^{ \pm}$ и $y_{2 n}^{ \pm}$единственных решений систем (43) с номерами $n=0,1$ непрерывны на полуоси $v \geqslant 0$ и убывают, как $o(1)$ и $o\left(v^{2 \nu}\right)$ при $v \rightarrow 0$. Таким образом, первый этап метода математической индукции завершен. Предположим, что при некотором целом $p>1$ все системы (43) с номерами $n \leqslant p$ однозначно разрешимы в классе непрерывных на полуоси $v \geqslant 0$ функций, удовлетворяющих соотношениям $y_{1 n}=o(1) y_{2 n}=o\left(v^{2 \nu}\right)$ при $v \rightarrow 0$. Теперь докажем, что система (43) с номером $n=p+1$ имеет единственное решение в том же классе функций. Благодаря предположенным выше свойствам компонент $y_{\text {in }}$ с $n \leqslant p$ все утверждения леммы 3 остаются в силе и в случае $n=p+1$. Следовательно, функции $r_{i n}^{ \pm}$при $n=p+1$ непрерывны на полуоси $v \geqslant 0$ и $r_{1 n}^{ \pm}=o(1)$, $r_{2 n}^{ \pm}=o\left(v^{2 \nu}\right)$ в пределе $v \rightarrow 0$. Этих свойств вполне достаточно для того, чтобы дословно повторить вывод всех формул (67)-(70) и их следствий, но уже в случае $n=p+1$ и таким образом доказать существование единственного и непрерывного на полуоси $v \geqslant 0$ решения системы (43) при любом ее номере $n=1,2, \ldots, m$.

Приступим к анализу оставшегося случая $2 \lambda=-1$.

ТЕОРема 5. Пусть $2 \lambda=-1$, и пусть при $n=0$ выполнены условия (8), а при $n=1,2, \ldots, m$ выполнены условия (1) u (2) с $2 \beta>3(m+1)$. Тогда система (43) имеет единственное непрерывное на полуоси $v \geqslant 0$ решение $\left\{y_{1 n}^{ \pm}, y_{2 n}^{ \pm}\right\}$, причем такое, umo $y_{1 n}^{ \pm}(v)=\delta_{0, n}+o\left(\ln ^{3} v\right) \ln ^{3} v$, a $y_{2 n}^{ \pm}(v)=o\left(\ln ^{2} v\right) \ln v n p u v \rightarrow 0$.

ДокАЗАТЕЛЬСтво отличается от доказательств леммы 3 и теорем 3 и 4 только тем, что вместо асимптотик (47)-(49) функций $f_{\lambda n}^{ \pm}$и $\theta_{\lambda n}^{ \pm}$при $2 \lambda>-1$ используются соответствующие асимптотики (47), (49) и (50) при $2 \lambda=-1$, а для оценки произведения $\left|p_{1}^{ \pm} p_{2}^{ \pm}\right|$вместо неравенства (53) применяется неравенство (58).

Теперь можно сформулировать и доказать итоговую теорему.

Теорема 6. Пусть $2 \lambda \geqslant-1$, и пусть при $n=0$ выполнены условия (8), а при $n=1,2, \ldots, m-$ условия (1) и (2) с $2 \beta>3(m+1)$. Тогда задача Коши (10), (11) имеет непрерывное на полуоси $v \geqslant 0$ решение $\left\{c_{n}^{ \pm}, s_{n}^{ \pm}\right\}$, причем такое, что

$$
\begin{aligned}
& c_{n}^{ \pm}(v)=\delta_{0, n}+o\left(\ln ^{3} v\right) \ln ^{3} v, \quad s_{n}^{ \pm}(v)=o\left(\ln ^{2} v\right) \ln v, \quad 2 \lambda=-1, \\
& c_{n}^{ \pm}(v)=\delta_{0, n}+o(1), \quad s_{n}^{ \pm}(v)=o\left(v^{2 \lambda+4}\right), \quad 2 \lambda>-1, \\
& v \rightarrow 0 \text {. }
\end{aligned}
$$

На полуоси $v \geqslant 0$ компоненты $c_{0}^{+} u s_{0}^{+}$или $c_{0}^{-} u s_{0}^{-}$не имеют общих нулей. 
ДокАзАтЕльство несложное. Существование, единственность и непрерывность функций $c_{n}^{ \pm}$и $s_{n}^{ \pm}$на полуоси $v \geqslant 0$ следуют из однозначности и непрерывности представлений (39) этих функций через функции $y_{1 n}^{ \pm}$и $y_{2 n}^{ \pm}$и теорем $3-5$. Благодаря тем же представлениям в пределе $v \rightarrow 0$ функции $c_{n}^{ \pm}$и $s_{n}^{ \pm}$ведут себя так же, как функции $y_{1 n}^{ \pm}$и $y_{2 n}^{ \pm}$.

Осталось доказать, что при любом $\lambda \geqslant-1 / 2$ на полуоси $v \geqslant 0$ не существует точки, в которой обе функции $c_{0}^{+}$и $s_{0}^{+}$или $c_{0}^{-}$и $s_{0}^{-}$равны нулю. Ключевыми для доказательства от противного будут два факта: в точке $v=0$ функция $c_{0}^{ \pm}$непрерывна справа и равна единице.

Предположим противное: пусть $c_{0}^{ \pm}(v)=0$ и $s_{0}^{ \pm}(v)=0$ в точке $v=\infty$. Эти равенства используем как начальные условия для уравнений (35) при $n=0$ в области $v>0$. Исследуем сформулированную таким образом задачу Коши. Подстановкой

$$
t=1-e^{-v}, \quad c_{0}^{ \pm}(v)=x_{1}^{ \pm}(t), \quad s_{0}^{ \pm}(v)=x_{2}^{ \pm}(t)
$$

сведем ее к линейной относительно неизвестных функций $x_{1}^{ \pm}$и $x_{2}^{ \pm}$задаче Коши на отрезке $0 \leqslant t \leqslant 1$. Запишем такую задачу в виде системы двух уравнений

$$
\partial_{t} x_{i}^{ \pm}(t)=g_{i}^{ \pm}\left(t, x_{1}^{ \pm}, x_{2}^{ \pm}\right), \quad i=1,2, \quad t \in[0,1]
$$

с тривиальными начальными условиями $x_{i}^{ \pm}(t)=0, i=1,2$, в точке $t=1$.

На полуинтервале $0<t \leqslant 1$ функции $g_{1}^{ \pm}$и $g_{2}^{ \pm}$непрерывны по переменной $t$ и имеют непрерывные частные производные по переменным $x_{1}^{ \pm}$и $x_{2}^{ \pm}$. Следовательно, выполнены оба достаточных условия теоремы Пикаро [8]. По этой теореме на полуинтервале $0<t \leqslant 1$ обсуждаемая задача Коши для функций $x_{i}^{ \pm}, i=1,2$, имеет единственное и при этом непрерывное решение. Очевидно, что им является тривиальное решение $x_{i}^{ \pm}(t) \equiv 0, t \in(0,1], i=1,2$. Так как $c_{0}^{ \pm}(v)=x_{1}^{ \pm}(t)$, получаем, что $c_{0}^{ \pm}(v)=0$ при любом $v>0$, но при этом $c_{0}^{ \pm}(v)=1$ в точке $v=0$. Следовательно, в этой точке функция $c_{0}^{ \pm}$терпит разрыв первого рода, но эта функция непрерывна в точке $v=0$ справа. Полученное противоречие означает, что ранее высказанное предположение $c_{0}^{ \pm}(\infty)=0$ и $s_{0}^{ \pm}(\infty)=0$ неверно. Аналогичным образом нетрудно показать, что не существует конечного значения переменной $v$, при котором обе компоненты $c_{0}^{ \pm}$и $s_{0}^{ \pm}$обращаются в нуль. Теорема доказана.

Сормулируем некоторые важные следствия леммы 3 и теорем 4-6. Условия (1) и $(2)$ с $2 \beta>3(m+1)$ являются достаточными для того, чтобы все функции $d_{i n}^{ \pm}, r_{i n}^{ \pm}$, $y_{i n}^{ \pm}, c_{i n}^{ \pm}$и $s_{i n}^{ \pm}$с номерами $n=1,2, \ldots, m$ оказались непрерывными на полуоси $v \geqslant 0$ и, следовательно, ограниченными в точке $v=\infty$. Как пояснялось в лемме 3 , при условиях (1) и (2) в случае $2 \beta \leqslant 3(m+2)$ интегралы $d_{1 n}^{ \pm}$и $d_{2 n}^{ \pm}, n=m+1$, могут расходиться в пределе $v \rightarrow \infty$. Таким образом, согласно доказательствам теорем 4-6 в этом случае все функции $y_{i n}^{ \pm}, c_{i n}^{ \pm}$и $s_{i n}^{ \pm}$с номером $n=m+1$, вообще говоря, не принимают определенных значений в точке $v=\infty$.

\section{5. НИЗКОЭНЕРГЕТИЧЕСКИЕ АСИМПТОТИКИ}

В настоящем разделе решим следующую задачу: при любом значении квантового числа $\lambda$ вывести и исследовать низкоэнергетические (при $q \rightarrow 0$ ) асимптотики редуцированных амплитудных функций $c^{ \pm}$и $s^{ \pm}$, функции эффективного радиуса, фазы $\delta_{\lambda}^{ \pm}$, сечения $\sigma_{\lambda}^{ \pm}$и радиальной волновой функции $u_{\lambda}^{ \pm}$ядерно-кулоновского рассеяния. Для решения поставленной задачи используем доказанные в теореме 6 свойства компонент $c_{n}^{ \pm}$и $s_{n}^{ \pm}$. 
5.1. Асимптотики редуцированных амплитудных функций. Сформулируем теорему о низкоэнергетических асимптотиках функций $c^{ \pm}$и $s^{ \pm}$.

ТеОрема 7. Пусть $2 \lambda \geqslant-1, m \geqslant 1$, выполнены условия (1) и (2) с $2 \beta>3(m+1)$. Тогда имеют место соотношения

$$
\begin{aligned}
& c^{ \pm}(v, q)=\sum_{n=0}^{m-1} q^{2 n} c_{n}^{ \pm}(v)+q^{2 m} C^{ \pm}(v, q), \\
& s^{ \pm}(v, q)=\sum_{n=0}^{m-1} q^{2 n} s_{n}^{ \pm}(v)+q^{2 m} S^{ \pm}(v, q),
\end{aligned}
$$

в которых функции $C^{ \pm}$и $S^{ \pm}$являются непрерывными на полуоси $v \geqslant 0$ и сходятся $\kappa$ компонентам $c_{m}^{ \pm}$u $s_{m}^{ \pm}$в пределе $q \rightarrow 0$.

ДокАЗАтельство. В задаче Коши (27), (28) заменим функции $c^{ \pm}$и $s^{ \pm}$соответствующими суммами (71). Затем, используя системы уравнений (35) с номерами $n<m$, приведем подобные слагаемые. В результате на интервале $v>0$ получим систему уравнений

$$
\begin{aligned}
& \partial_{v} C^{ \pm}(v, q)=v^{3} V(v)\left\{\left[C^{ \pm}(v, q) f_{\lambda}^{ \pm}(v, q)+S^{ \pm}(v, q) \theta_{\lambda}^{ \pm}(v, q)\right] \theta_{\lambda}^{ \pm}(v, q)+R_{1}^{ \pm}(v, q)\right\} \\
& \partial_{v} S^{ \pm}(v, q)=-v^{3} V(v)\left\{\left[C^{ \pm}(v, q) f_{\lambda}^{ \pm}(v, q)+S^{ \pm}(v, q) \theta_{\lambda}^{ \pm}(v, q)\right] f_{\lambda}^{ \pm}(v, q)+R_{2}^{ \pm}(v, q)\right\}
\end{aligned}
$$

с начальными условиями

$$
\left.C^{ \pm}(v, q)\right|_{v=0}=0,\left.\quad S^{ \pm}(v, q)\right|_{v=0}=0 .
$$

Слагаемые $R_{1}^{ \pm}$и $R_{2}^{ \pm}$системы (72) содержат компоненты $c_{n}^{ \pm}, s_{n}^{ \pm}$с номерами $n<m$ и функции $A_{\lambda}^{ \pm}$и $B_{\lambda}^{ \pm}$, которые порождаются следующими разбиениями рядов (33):

$$
\begin{aligned}
& f_{\lambda}^{ \pm}(v, q)=\sum_{n=0}^{m-1} q^{2 n} f_{\lambda n}^{ \pm}(v)+q^{2 m} A_{\lambda}^{ \pm}(v, q), \\
& \theta_{\lambda}^{ \pm}(v, q)=\sum_{n=0}^{m-1} q^{2 n} \theta_{\lambda n}(v)+q^{2 m} B_{\lambda}^{ \pm}(v, q) .
\end{aligned}
$$

По теореме 6 все компоненты $c_{n}^{ \pm}$и $s_{n}^{ \pm}$с номерами $n<m$ непрерывны на полуоси $v \geqslant 0$. Как показано в работе [6], ряды (33) сходятся равномерно. Следовательно, этим же свойством обладают и их остатки $q^{2 m} A_{\lambda}^{ \pm}$и $q^{2 m} B_{\lambda}^{ \pm}$, содержащие компоненты $f_{\lambda n}^{ \pm}$и $\theta_{\lambda n}^{ \pm}$с $n \geqslant m$. Такие компоненты имеют асимптотики (47)-(50), поэтому при ограничениях (1) и (2) на потенциал $V($ при $2 \beta>3(m+1))$ эти компоненты интегрируемы на любом отрезке $[0, v], v>0$, с весом $t^{3} V(t)$. В силу перечисленных выше свойств функций $c_{n}^{ \pm}, s_{n}^{ \pm}, f_{\lambda n}^{ \pm}, \theta_{\lambda n}^{ \pm}$и остатков $q^{2 m} A_{\lambda}^{ \pm}$и $q^{2 m} B_{\lambda}^{ \pm}$произведения $t^{3} V(t) R_{1}^{ \pm}(t, q)$ и $t^{3} V(t) R_{2}^{ \pm}(t, q)$ интегрируемы на любом отрезке $[0, v], v>0$, и при любом $q \geqslant 0$. Это свойство слагаемых $R_{1}^{ \pm}$и $R_{2}^{ \pm}$является достаточным для доказательства однозначной разрешимости задачи Коши (72), (73) в классе функций, непрерывных на полуоси $v \geqslant 0$.

Поясним ключевые этапы дальнейшего анализа этой задачи. Сначала вводится интеграл

$$
B^{ \pm}(v, q) \equiv \int_{0}^{v} t^{3} V(t) f_{\lambda}^{ \pm}(t) \theta_{\lambda}^{ \pm}(t) d t
$$


Затем подстановкой

$$
C^{ \pm}(v)=z_{1}^{ \pm}(v) e^{B^{ \pm}(v, q)}, \quad S^{ \pm}(v)=z_{2}^{ \pm}(v) e^{-B^{ \pm}(v, q)}
$$

задача Коши (72), (73) сводится к недиагональной системе интегральных уравнений для искомых функций $z_{1}^{ \pm}$и $z_{2}^{ \pm}$. Из этой системы после одной итерации получается диагональная система интегральных уравнений. Она исследуется методом последовательных приближений по схеме, подробно изложенной в работе [7]. Таким образом доказывается, что задача Коши (72), (73) имеет единственное решение $\left\{C^{ \pm}, S^{ \pm}\right\}$, компоненты которого непрерывны при $v \geqslant 0$ и $q \geqslant 0$. Поэтому и в уравнениях (72), и в начальных условиях (73) можно перейти к пределу $q \rightarrow 0$. В итоге получается задача Коши для функций $C^{ \pm}(v, 0)$ и $S^{ \pm}(v, 0)$. Заменой обозначений $C^{ \pm}(v, 0) \rightarrow c_{m}^{ \pm}(v)$ и $S^{ \pm}(v, 0) \rightarrow s_{m}^{ \pm}(v)$ эта задача сводится к уже исследованной задаче Коши $(35),(36)$. Следовательно, $C^{ \pm}(v, q) \rightarrow c_{m}^{ \pm}(v)$ и $S^{ \pm}(v, q) \rightarrow s_{m}^{ \pm}(v)$ при $q \rightarrow 0$.

Осталось выявить смысл разложений (71). Для этого символами $T_{1}^{ \pm}$и $T_{2}^{ \pm}$обозначим суммы функций $q^{2 n} c_{n}^{ \pm}$и $q^{2 n} s_{n}^{ \pm}(v)$ с номерами $n=0,1, \ldots, m-1$. Разбиения (71) означают, что на всей полуоси $v \geqslant 0$ и, в частности, в ее бесконечно удаленной точке $v=\infty$ суммы $T_{1}^{ \pm}$и $T_{2}^{ \pm}$являются низкоэнергетическими приближениями функций $c^{ \pm}$и $s^{ \pm}$с абсолютной точностью $O\left(q^{2 m}\right)$. Этим пояснением завершим доказательство теоремы.

Теперь обсудим построение низкоэнергетических приближений нормировочного множителя $N^{ \pm}$, фазы $\delta_{\lambda}^{ \pm}$, сечения $\sigma_{\lambda}^{ \pm}$и радиальной волновой функции $u_{\lambda}^{ \pm}$рассеяния квантовой частицы $p_{1}$ в состоянии $|\lambda, q\rangle$. Функции $N_{m}^{ \pm}(q), \operatorname{tg} \delta_{\lambda m}^{ \pm}(q), \sigma_{\lambda m}^{ \pm}(q)$ и $u_{\lambda m}^{ \pm}(v, q)$ определим как правые части соответствующих равенств $(29)-(32)$, в которых функции $c^{ \pm}$и $s^{ \pm}$заменены их приближениями $T_{1}^{ \pm}$и $T_{2}^{ \pm}$. По определению функции $N_{m}^{ \pm}, \operatorname{tg} \delta_{\lambda m}^{ \pm}, \sigma_{\lambda m}^{ \pm}$и $u_{\lambda m}^{ \pm}$содержат только известные функции $C_{\lambda}, h, f_{\lambda n}$, $\theta_{\lambda n}$ и компоненты $c_{n}^{ \pm}, s_{n}^{ \pm}$с номерами $n \leqslant m-1$ и являются низкоэнергетическими приближениями соответствующих функций $N^{ \pm}, \operatorname{tg} \delta_{\lambda}^{ \pm}$и $\sigma_{\lambda}^{ \pm}$с абсолютной точностью порядка $O\left(q^{2 m}\right)$. Следовательно, задача о построении низкоэнергетических приближений $N_{m}^{ \pm}, \operatorname{tg} \delta_{\lambda m}^{ \pm}, \sigma_{\lambda m}^{ \pm}$и $u_{\lambda m}^{ \pm}$сводится к вычислению конечного числа компонент $c_{n}^{ \pm}$ и $s_{n}^{ \pm}, n=0,1, \ldots, m-1$. В этом заключается первое преимущество предлагаемого асимптотического метода. Второе преимущество состоит в том, что все такие компоненты удовлетворяют конечной, энергонезависимой и рекуррентной цепочке задач Коши (35), (36), численный анализ которых довольно прост.

В следующих пп. 5.2-5.4 для анализа особенностей низкоэнергетических приближений функции эффективного радиуса, фазы $\delta_{\lambda}^{ \pm}$, сечения $\sigma_{\lambda}^{ \pm}$и нормировочного множителя $N^{ \pm}$нам потребуются свойства предельных при $v \rightarrow \infty$ значений компонент $c_{0}^{ \pm}$и $s_{0}^{ \pm}$.

Покажем, что $c_{0}^{+}(v) \neq 0$ при любом $v \geqslant 0$. По теореме 1 при любом $q>0$ амплитудные функции $c$ и $s$ не имеют общих нулей. Следовательно, верно соотношение

$$
|c(\rho, \eta)|+|s(\rho, \eta)| \neq 0, \quad \rho \geqslant 0 .
$$

Заменим в нем функции $c$ и $s$ правыми частями равенств $(22)$, в которых представим функции $c^{ \pm}$и $s^{ \pm}$рядами (34). По теореме 7 такие ряды сходятся равномерно, поэтому в полученном соотношении можно перейти к пределу $q \rightarrow 0$ и, используя асимптотики (17) и (25) функций $C_{\lambda}^{2}$ и $h$, вывести два неравенства:

$$
\left|c_{0}^{+}(v)\right| \neq 0, \quad\left|c_{0}^{-}(v)\right|+\pi\left|s_{0}^{-}(v)\right| \neq 0, \quad v \geqslant 0 .
$$


Первое из них означает, что компонента $c_{0}^{+}$всегда отлична от нуля, а второе подтверждает утверждение теоремы 6 об отсутствии общих нулей у компонент $c_{0}^{-}$и $s_{0}^{-}$.

Сформулируем важное следствие этой теоремы и доказанного выше неравенства $c_{0}^{+}(v) \neq 0$ для $v \geqslant 0$. При $v=\infty$ возможны три случая. Случай $1: c_{0}^{ \pm}(v) \neq 0$ и $s_{0}^{ \pm}(v) \neq 0$. Случай $2: s_{0}^{ \pm}(v)=0$, но $c_{0}^{ \pm}(v) \neq 0$. Случай $3: c_{0}^{-}(v)=0$, но $s_{0}^{-}(v) \neq 0$.

В следующих пп. 5.2-5.4 для полноты изложения мы исследуем все эти случаи. При этом будем предполагать, что потенциал $V$ удовлетворяет условиям (1) и условиям (2), в которых $2 \beta>3(m+1)$, а $m=3$. При таких условиях по теореме 6 компоненты $c_{n}^{ \pm}$и $s_{n}^{ \pm}, n=0,1,2,3$, ограничены в точке $v=\infty$, а по теореме 7 верны представления (71) с $m=3$. В пп. 5.5 выясним, какие из полученных в пп. 5.2-5.4 асимптотических соотношений остаются в силе при более слабых условиях (2) с параметром $2 \beta>3(m+1)$, но при $m<3$.

5.2. Функция эффективного радиуса и ее асимптотика. Для данного состояния $|q, \lambda\rangle$ рассеяния квантовой частицы $p_{1}$ определим функцию эффективного радиуса как функцию волнового числа $q$, удовлетворяющую трем условиям: она должна содержать котангенс фазы $\delta_{\lambda}^{ \pm}(q)$ и иметь низкоэнергетическую (при $q \rightarrow 0$ ) асимптотику в виде ряда Маклорена по четным степеням аргумента $q$, при этом старшее слагаемое этого ряда должно быть конечной, но ненулевой константой.

Приступим к построению функции эффективного радиуса для состояния $|q, \lambda\rangle$. Введем вспомогательную функцию

$$
K^{ \pm}(v, q) \equiv(\nu !)^{2} q^{2 \lambda+1} C_{\lambda}^{2}( \pm|\eta|) \operatorname{ctg} \delta_{\lambda}^{ \pm}(v, q)-h(q)
$$

где функции $C_{\lambda}( \pm|\eta|)$ и $h(q)$ заданы равенствами (16) и $(20),(23),(24)$, а фазовая функция $\delta_{\lambda}^{ \pm}(v, q)$ представляется через функции $c^{ \pm}(v, q)$ и $s^{ \pm}(v, q)$ формулой (30). Используя эту формулу и представления (71) с $m=3$, запишем функцию $K^{ \pm}(v, q)$ в виде

$$
K^{ \pm}(v, q)=\frac{c^{ \pm}(v, q)}{s^{ \pm}(v, q)}=\frac{c_{0}^{ \pm}(v)+q^{2} c_{1}^{ \pm}(v)+q^{4} c_{2}^{ \pm}(v)+q^{6} C^{ \pm}(v, q)}{s_{0}^{ \pm}(v)+q^{2} s_{1}^{ \pm}(v)+q^{4} s_{2}^{ \pm}(v)+q^{6} S^{ \pm}(v, q)} .
$$

Далее для краткости символами $A(q)$ и $B$ будем обозначать значения функций $A(v, q)$ и $B(v)$ в бесконечно удаленной точке $v=\infty$. Согласно теоремам 2 и 6 функции $c^{ \pm}(v, q)$ и $s^{ \pm}(v, q)$ и все их компоненты $c_{n}^{ \pm}(v), s_{n}^{ \pm}(v), n \leqslant m=3$, непрерывны в бесконечно удаленной точке $v=\infty$ и, следовательно, принимают в этой точке вполне определенные и конечные значения $c^{ \pm}(q), s^{ \pm}(q)$ и $c_{n}^{ \pm}, s_{n}^{ \pm}$. Поэтому в соотношениях (75) и (76) можно перейти к пределу $v \rightarrow \infty$ и таким образом получить представление функции $K(q)$ через фазу рассеяния $\delta_{\lambda}^{ \pm}(q)$ и представление этой же функции через предельные значения функций $c^{ \pm}(v, q)$ и $s^{ \pm}(v, q)$ и их компонент $c_{n}^{ \pm}(v), s_{n}^{ \pm}(v)$ :

$$
\begin{aligned}
K^{ \pm}(q) & =(\nu !)^{2} q^{2 \lambda+1} C_{\lambda}^{2}( \pm|\eta|) \operatorname{ctg} \delta_{\lambda}^{ \pm}(q)-h(q)=\frac{c^{ \pm}(q)}{s^{ \pm}(q)}= \\
& =\frac{c_{0}^{ \pm}+q^{2} c_{1}^{ \pm}+q^{4} c_{2}^{ \pm}+q^{6} C^{ \pm}(q)}{s_{0}^{ \pm}+q^{2} s_{1}^{ \pm}+q^{4} s_{2}^{ \pm}+q^{6} S^{ \pm}(q)} .
\end{aligned}
$$

Из равенств $(76)$ и $(77)$ следует, что $K(v, q)$ и $K(q)$ являются четными функциями переменной $q \geqslant 0$. Исследуем эти равенства в трех случаях. 
СлучАй 1. Пусть в точке $v=\infty$ функции $c_{0}^{ \pm}(v)$ и $s_{0}^{ \pm}(v)$ не равны нулю $\left(c_{0}^{ \pm} \neq\right.$ $\left.0, s_{0}^{ \pm} \neq 0\right)$. Запишем функцию $K^{ \pm}(v, q)$ в виде ряда Маклорена по аргументу $q$ с остаточным слагаемым $P^{ \pm}(v, q)=O\left(q^{6}\right)$ :

$$
K^{ \pm}(v, q)=\sum_{n=0}^{2} q^{2 n} M_{n}^{ \pm}(v, q)+P^{ \pm}(v, q)
$$

В этом выражении

$$
M_{0}^{ \pm}(v)=\frac{c_{0}^{ \pm}(v)}{s_{0}^{ \pm}(v)}, \quad M_{1}(v)=\frac{c_{1}^{ \pm}(v)-s_{1}^{ \pm}(v) M_{0}^{ \pm}(v)}{s_{0}^{ \pm}(v)}
$$

а каждая функция $M_{n}^{ \pm}(v), n=0,1,2$, представляется конечной суммой дробей, знаменатели которых - целые степени $\left(s_{0}^{ \pm}(v)\right)^{p}$ с $p \leqslant 2 n$, а числители выражаются через компоненты $c_{m}^{ \pm}(v)$ и $s_{m}^{ \pm}(v)$ с номерами $m \leqslant n$. Поэтому обсуждаемый ряд Маклорена (78) сходится при любом значении аргумента $v$, отличном от нуля функции $s_{0}^{ \pm}(v)$. В рассматриваемом случае эта функция не равна нулю в точке $v=\infty$. Следовательно, функции $P^{ \pm}(v, q)$ и $M_{n}^{ \pm}(v)$ для $n=0,1,2$, а следовательно, функции $a_{1}^{ \pm}(v)$, и $r_{1}^{ \pm}(v)$, заданные формулами

$$
\begin{aligned}
& a_{1}^{ \pm}(v) \equiv-\frac{1}{M_{0}^{ \pm}(v)}=-\frac{s_{0}^{ \pm}(v)}{c_{0}^{ \pm}(v)} \\
& r_{1}^{ \pm}(v) \equiv 2 M_{1}^{ \pm}(v)=\frac{2}{s_{0}^{ \pm}(v)}\left[c_{1}^{ \pm}(v)-s_{1}^{ \pm}(v) \frac{c_{0}^{ \pm}(v)}{s_{0}^{ \pm}(v)}\right]
\end{aligned}
$$

ограничены в пределе $v \rightarrow \infty$, при этом функция $a_{1}^{ \pm}(v)$ в $v=\infty$ принимает ненулевое значение. Поэтому и в определении (76) функции $K(v, q)$, и во всех соотношениях (78)-(80) можно положить $v=\infty$. В результате получится следующее представление предела $K^{ \pm}(q)$ исследуемой функции $K^{ \pm}(v, q)$ :

$$
K^{ \pm}(q)=(\nu !)^{2} q^{2 \lambda+1} C_{\lambda}^{2}( \pm|\eta|) \operatorname{ctg} \delta_{\lambda}^{ \pm}(q)-h(q)=\frac{c^{ \pm}(q)}{s^{ \pm}(q)}=-\frac{1}{a_{1}^{ \pm}}+\frac{1}{2} r_{1}^{ \pm} q^{2}+t_{1}^{ \pm}(q)
$$

В этом представлении $a_{1}^{ \pm} \neq 0$ и $r_{1}^{ \pm}$- предельные (при $v \rightarrow \infty$ ) значения функций $a_{1}^{ \pm}(v)$ и $r_{1}^{ \pm}(v)$, а остаточное слагаемое $t_{1}^{ \pm}(q)$ убывает, как $O\left(q^{4}\right)$ при $q \rightarrow 0$. Поэтому в этом пределе обсуждаемое представление является низкоэнергетической асимптотикой функции $K^{ \pm}(q)$. Функцию $K_{1}^{ \pm}(q)=K^{ \pm}(q)$ и коэффициенты $a_{1}^{ \pm}$и $r_{1}^{ \pm}$ назовем функцией эффективного радиуса, длиной рассеяния и эффективным радиусом.

Функция эффективного радиуса для двумерного рассеяния суперпозицией потенциалов $V^{\mathrm{c}}+V^{\mathrm{s}}$ впервые исследована в работе [1], но только в случае $2 \lambda=-1$. Полученное в этой работе представление данной функции совпадает с формулой (81), если в ней положить $\lambda=-1 / 2$.

Теперь поясним первый способ вычисления функций $K^{ \pm}(v, q), a_{1}^{ \pm}(v)$ и $r_{1}^{ \pm}(v)$. Сначала необходимо решить задачи Коши $(27),(28)$ и $(35),(36)$ для функций $c^{ \pm}(v, q)$ и $s^{ \pm}(v, q)$ и их компонент $c_{n}^{ \pm}(v)$ и $s_{n}^{ \pm}(v), n=0,1$, а затем воспользоваться представлениями (76) и (80). 
Предложим другой способ вычисления функций $K(v, q), a_{1}^{ \pm}(v)$ и $r_{1}^{ \pm}(v)$, в котором не требуется решение упомянутых выше задач Коши.

Начнем с функции $K(v, q)=c^{ \pm}(v, q) / s^{ \pm}(v, q)$. Согласно условиям (28) в точке $v=0$ функция $s^{ \pm}(v, q)$ равна нулю. Следовательно, в этой точке функция $K(v, q)$ неограничена. Поэтому вместо нее удобнее использовать функцию $L^{ \pm}(v, q)$, определенную равенствами

$$
L^{ \pm}(v, q)=\frac{1}{K^{ \pm}(v, q)}=\frac{s^{ \pm}(v, q)}{c^{ \pm}(v, q)}
$$

и равную нулю в точке $v=0$. Выразим производную $\partial_{v} L^{ \pm}$через производные $\partial_{v} c^{ \pm}$ и $\partial_{v} s^{ \pm}$, которые заменим правыми частями уравнений $(27)$, и в результате получим следующую нелинейную задачу:

$$
2 \partial_{v} L^{ \pm}(v, q)=v^{3} V(v)\left[f_{\lambda}^{ \pm}(v, q)-L^{ \pm}(v, q) \theta_{\lambda}^{ \pm}(v, q)\right]^{2}, \quad v>0 ;\left.\quad L(v, q)\right|_{v=0}=0 .
$$

Исследуем функцию $a_{1}^{ \pm}(v)=-s_{0}^{ \pm}(v) / c_{0}(v)$. Согласно начальным условиям (36) в точке $v=0$ числитель этой дроби равен нулю. Поэтому $a_{1}^{ \pm}(v)=0$, если $v=0$. Продифференцируем определение (80) функции $a_{1}^{ \pm}(v)$ по переменной $v$, а затем заменим производные $\partial_{v} c_{0}^{ \pm}$и $\partial_{v} s_{0}^{ \pm}$правыми частями уравнений (35) и таким образом покажем, что функция $a_{1}^{ \pm}(v)$ подчиняется нелинейной задаче

$$
\partial_{v} a_{1}^{ \pm}(v)=v^{3} V(v)\left[f_{\lambda 0}^{ \pm}(v)-a_{1}^{ \pm}(v) \theta_{\lambda 0}^{ \pm}(v)\right]^{2}, \quad v>0 ;\left.\quad a_{1}^{ \pm}(v)\right|_{v=0}=0 .
$$

Теперь сформулируем и решим задачу Коши для функции $r_{1}^{ \pm}(v)$. Если в ее определении (80) устремить аргумент $v$ к нулю и заменить все компоненты $c_{n}^{ \pm}(v)$ и $s_{n}^{ \pm}(v), n=0,1$, их асимптотиками, упомянутыми в теореме 6 , то получится начальное условие $r_{1}^{ \pm}(v)=0$ при $v=0$. Продифференцируем определение (80) функции $r_{1}^{ \pm}(v)$ по переменной $v$. В полученном равенстве заменим производные $\partial_{v} c_{n}^{ \pm}$ и $\partial_{v} s_{n}^{ \pm}, n=0,1$, правыми частями соответствующих уравнений (35), а затем, используя формулы (80), выразим все комбинации компонент $c_{n}^{ \pm}(v)$ и $s_{n}^{ \pm}(v), n=0,1$, через функции $a_{1}^{ \pm}(v)$ и $r_{1}^{ \pm}(v)$. В итоге получим следующую задачу Коши:

$$
\partial_{v} r_{1}^{ \pm}(v)+\xi^{ \pm}(v)\left[\left(r_{1}^{ \pm}(v)-\phi^{ \pm}(v)\right]=0, \quad v>0 ;\left.\quad r_{1}^{ \pm}(v)\right|_{v=0}=0,\right.
$$

где по определению

$$
\xi^{ \pm}(v) \equiv 2 v^{3} V(v) f_{\lambda 0}^{ \pm}(v)\left[\frac{f_{\lambda 0}^{ \pm}(v)}{a_{1}^{ \pm}(v)}-\theta_{\lambda 0}^{ \pm}\right], \quad \phi^{ \pm}(v) \equiv \frac{2}{f_{\lambda 0}^{ \pm}(v)}\left[\frac{f_{\lambda 1}^{ \pm}(v)}{a_{1}^{ \pm}(v)}-\theta_{\lambda 1}^{ \pm}(v)\right] .
$$

Единственное решение такой задачи Коши имеет вид

$$
r_{1}^{ \pm}(v)=\frac{1}{z^{ \pm}(v)} \int_{0}^{v} \partial_{t} z^{ \pm}(t) \phi^{ \pm}(t) d t, \quad z^{ \pm}(v) \equiv \exp \left(\int_{0}^{v} \xi^{ \pm}(t) d t\right),
$$

и поэтому является интегральным представлением функции $r_{1}^{ \pm}(v)$ через $a_{1}^{ \pm}(v)$.

СлучАй 2. Предположим, что в точке $v=\infty$ функция $s_{0}^{ \pm}(v)$ обращается в нуль, а функция $s_{1}^{ \pm}(v)$ принимает ненулевое значение $s_{1}^{ \pm}$. По теореме 6 функция $c_{0}^{ \pm}(v)$ отлична от нуля при $v=\infty$. Итак, $s_{0}^{ \pm}=0, s_{1}^{ \pm} \neq 0$ и $c_{0}^{ \pm} \neq 0$. Следовательно, длина рассеяния $a_{1}^{ \pm}=-s_{0}^{ \pm} / c_{0}^{ \pm}$равна нулю, и поэтому необходимо переопределить 
функцию эффективного радиуса. Для этого сначала рассмотрим представление функции $K(q)$ в виде дроби (77), содержащей предельные значения компонент $c_{n}^{ \pm}(v)$ и $s_{n}^{ \pm}(v)$. Так как $s_{0}^{ \pm}=0, s_{1}^{ \pm} \neq 0$ и $c_{0}^{ \pm} \neq 0$, произведение $K_{2}^{ \pm} \equiv q^{2} K^{ \pm}(q)$ при $q \rightarrow 0$ равно конечной и ненулевой константе. Поэтому в рассматриваемом случае такое произведение мы считаем функцией эффективного радиуса. Представим ее формулами

$$
\begin{aligned}
K_{2}^{ \pm}(q) \equiv \lim _{v \rightarrow \infty} q^{2} K(v, q) & =(\nu !)^{2} q^{2 \lambda+3} C_{\lambda}^{2}( \pm|\eta|) \operatorname{ctg} \delta_{\lambda}^{ \pm}(q)-q^{2} h(q)= \\
& =-\frac{1}{a_{2}^{ \pm}}+\frac{1}{2} q^{2} r_{2}^{ \pm}+t_{2}^{ \pm}(q),
\end{aligned}
$$

где $t_{2}^{ \pm}(q)=O\left(q^{4}\right)$ при $q \rightarrow 0$, а длина рассеяния $a_{2}^{ \pm}$и эффективный радиус $r_{2}^{ \pm}$ определяются соответствующими пределами:

$$
a_{2}^{ \pm} \equiv-\lim _{v \rightarrow 0} \frac{s_{1}^{ \pm}(v)}{c_{0}^{ \pm}(v)}, \quad r_{2}^{ \pm} \equiv \lim _{v \rightarrow 0} \frac{2}{s_{1}^{ \pm}(v)}\left[c_{1}^{ \pm}(v)+\frac{s_{2}^{ \pm}(v)}{a_{2}^{ \pm}(v)}\right] .
$$

СлУчАй 3. Пусть в точке $v=\infty$ функция $c_{0}^{-}(v)$ обращается в нуль, но функция $c_{1}^{-}(v)$ принимает ненулевое значение $c_{1}^{-}$. Тогда по теореме $6 s_{0}^{-} \equiv s_{0}^{-}(v) \neq 0$ при $v=\infty$. Итак, $c_{0}^{-}=0, c_{1}^{-} \neq 0$ и $s_{0}^{-} \neq 0$. Следовательно, длина рассеяния $a_{1}^{-}=-s_{0}^{-} / c_{0}^{-}$не является конечным числом, и поэтому требуется переопределение функции эффективного радиуса. Для этого воспользуемся представлением функции $K(q)$ в виде дроби (77). Так как $c_{0}^{-}=0, c_{1}^{-} \neq 0$ и $s_{0}^{-} \neq 0$, произведение $K_{3}^{ \pm} \equiv q^{-2} K^{ \pm}(q)$ в пределе $q \rightarrow 0$ равно конечной и ненулевой константе. Поэтому в рассматриваемом случае такое произведение мы считаем функцией эффективного радиуса. Представим ее формулами

$$
\begin{aligned}
K_{3}^{-}(q) \equiv \lim _{v \rightarrow \infty} q^{-2} K^{-}(v, q) & =(\nu !)^{2} q^{2 \lambda-1} C_{\lambda}^{2}(-|\eta|) \operatorname{ctg} \delta_{\lambda}^{-}(v, q)-q^{-2} h(q)= \\
& =-\frac{1}{a_{3}^{-}}+\frac{1}{2} q^{2} r_{3}^{-}+t_{3}^{-}(q),
\end{aligned}
$$

где остаточное слагаемое $t_{3}^{-}(q)$ при $q \rightarrow 0$ убывает, как $O\left(q^{4}\right)$, а длина рассеяния $a_{3}^{ \pm}$ и эффективный радиус $r_{3}^{ \pm}$определены следующими предельными соотношениями:

$$
a_{3}^{-} \equiv-\lim _{v \rightarrow 0} \frac{s_{0}^{-}(v)}{c_{1}^{-}(v)}, \quad r_{3}^{-} \equiv \lim _{v \rightarrow 0} \frac{2}{s_{0}^{-}(v)}\left[c_{2}^{-}(v)+\frac{s_{1}^{-}(v)}{a_{3}^{-}(v)}\right] .
$$

5.3. Асимптотики парциальных фаз и сечений. Выведем и обсудим старшие слагаемые низкоэнергетических (при $q \rightarrow 0$ ) асимптотик кулоновской фазы $\delta_{\lambda}^{\mathrm{c}}( \pm|\eta|)$, ядерно-кулоновской фазы $\delta_{\lambda}^{ \pm}(q)=\delta_{\lambda}( \pm|\eta|)$ и трех слагаемых парциального сечения $\sigma_{\lambda}^{\mathrm{cs}}( \pm|\eta|)$ : кулоновского $\sigma_{\lambda}^{\mathrm{c}}( \pm|\eta|)$, ядерно-кулоновского $\sigma_{\lambda}^{ \pm}(q)=\sigma_{\lambda}( \pm|\eta|)$ и интерференционного $\sigma_{\lambda}^{\text {int }}( \pm|\eta|)$ сечений. Используем определения $(6),(7)$ таких сечений и определения (81)-(83) функций эффективного радиуса. Согласно правилу, принятому в разделе 2 , в символе $A( \pm|\eta|)$ или $B^{ \pm}(q)$ берем верхний знак в случае кулоновского отталкивания, а нижний - в случае кулоновского притяжения.

Начнем с обсуждения поведения фазы $\delta_{\lambda}^{\mathrm{c}}(\eta)$ и сечения $\sigma_{\lambda}^{\mathrm{c}}(\eta)$ кулоновского рассеяния при $q \rightarrow 0$. В этом пределе согласно формуле $(18)$ модули фаз $\delta_{\lambda}^{\mathrm{c}}(+|\eta|)$ и $\delta_{\lambda}^{\mathrm{c}}(-|\eta|)$ 
неограниченно возрастают, как $O\left(q^{-1}|\ln q|\right)$, поэтому сечения $\sigma_{\lambda}^{\mathrm{c}}(+|\eta|)$ и $\sigma_{\lambda}^{\mathrm{c}}(-|\eta|)$ быстро осциллируют, а их максимальные значения увеличиваются, как $O\left(q^{-1}\right)$.

Теперь рассмотрим те же три случая, что и в предыдущем пункте.

СлУчАй 1. Воспользуемся представлением (81) функции эффективного радиуса $K_{1}^{ \pm}(q) \equiv K(q)$. Сначала выразим тангенс фазы $\delta_{\lambda}^{ \pm}(q)$ через коэффициенты $a_{1}^{ \pm}$и $r_{1}^{ \pm}$ и функции $C_{\lambda}^{2}$ и $h$. Затем заменим эти функции их асимптотиками (17) и (25) и в итоге докажем, что

$$
\operatorname{tg} \delta_{\lambda}^{ \pm}(q)=-a_{1}^{ \pm}(\nu !)^{2} q^{\nu} C_{\lambda}^{2}( \pm \eta)\left[1+O\left(q^{2}\right)\right] \sim-\pi a_{1}^{ \pm}\left\{\begin{array}{c}
e^{-\pi / q} \\
1
\end{array}\right\}, \quad q \rightarrow 0 .
$$

Следовательно, в пределе $q \rightarrow 0$ фаза $\delta_{\lambda}^{+}(q)$ быстро убывает, а фаза $\delta_{\lambda}^{-}(q)$ сходится к своему значению $\delta_{\lambda}^{-}(0) \equiv-\operatorname{arctg}\left(\pi a_{1}^{-}\right)$в точке $q=0$. Поэтому верны приближения

$$
\sigma_{\lambda}^{+}(q) \sim \frac{|R|}{q} \varepsilon_{\lambda}\left(2 \pi a_{1}^{+}\right)^{2} e^{-2 \pi / q}, \quad \sigma_{\lambda}^{-}(q) \sim \frac{|R|}{q} \varepsilon_{\lambda} \frac{\left(2 \pi a_{1}^{-}\right)^{2}}{1+\left(\pi a_{1}^{-}\right)^{2}}, \quad q \rightarrow 0 .
$$

Следовательно, в пределе $q \rightarrow 0$ сечение $\sigma_{\lambda}^{-}$растет, как $O\left(q^{-1}\right)$, а сечение $\sigma_{\lambda}^{+}$быстро убывает и экспоненциально мало по сравнению с сечением $\sigma_{\lambda}^{\text {c }}$.

Из формул (18) и (84) следует, что $\left|\delta_{\lambda}^{\mathrm{c}}( \pm|\eta|)\right| \gg\left|\delta_{\lambda}^{ \pm}(q)\right|$ при $q \rightarrow 0$. Поэтому

$$
\sigma_{\lambda}^{\mathrm{int}}( \pm|\eta|) \sim 4 \frac{|R|}{q} \varepsilon_{\lambda} \sin \left[2 \delta_{\lambda}^{\mathrm{c}}( \pm|\eta|)\right]\left\{\begin{array}{c}
-\pi a_{1}^{ \pm} e^{-\pi / q} \\
\sin \delta_{\lambda}^{-}(0)
\end{array}\right\}, \quad q \rightarrow 0 .
$$

Следовательно, в пределе $q \rightarrow 0$ сечение $\sigma_{\lambda}^{\text {int }}(+|\eta|)$, осциллируя, сходится к нулю, сечение $\sigma_{\lambda}^{\text {int }}(-|\eta|)$ тоже осциллирует, но амплитуда его осцилляций возрастает, как $O\left(q^{-1}\right)$.

СлучАй 2. Используя определение (82) функции эффективного радиуса и те же приемы, что и в случае 1 , нетрудно вывести асимптотические (при $q \rightarrow 0$ ) соотношения

$$
\delta_{\lambda}^{ \pm}(q) \sim-\pi a_{2}^{ \pm} q^{2}\left\{\begin{array}{c}
e^{-\pi / q} \\
1
\end{array}\right\}, \quad \sigma_{\lambda}^{ \pm}(q) \sim\left(2 \pi a_{2}^{ \pm}\right)^{2}|R| \varepsilon_{\lambda} q^{3}\left\{\begin{array}{c}
e^{-2 \pi / q} \\
1
\end{array}\right\}
$$

а затем показать, что

$$
\sigma_{\lambda}^{\text {int }}( \pm|\eta|) \sim-4 \pi a_{1}^{ \pm}|R| q \varepsilon_{\lambda} \sin \left[2 \delta_{\lambda}^{\mathrm{c}}( \pm|\eta|)\right]\left\{\begin{array}{c}
e^{-\pi / q} \\
1
\end{array}\right\}, \quad q \rightarrow 0 .
$$

В данном случае в отличие от предыдущего фаза $\delta_{\lambda}^{-}$и сечения $\sigma_{\lambda}^{-}, \sigma_{\lambda}^{\text {int }}(-|\eta|)$ монотонно сходятся к нулю при $q \rightarrow 0$.

СлУчАй 3. В этом случае функция эффективного радиуса определяется соотношениями (83). Согласно формуле $(25)$ в первом из них $q^{-2} h(q)=-1 / 6+O\left(q^{2}\right)$. Поэтому при $q \rightarrow 0$

$$
\operatorname{tg} \delta_{\lambda}^{-}(q) \sim \psi(q) \equiv-\frac{6 \pi}{q^{2}} \frac{a_{3}^{-}}{6+a_{3}^{-}\left(1-3 q^{2} r_{3}^{-}\right)}, \quad \sigma_{\lambda}^{-}(q) \sim 4 \frac{|R|}{q} \varepsilon_{\lambda} \frac{\psi^{2}(q)}{1+\psi^{2}(q)}
$$


Следовательно, в пределе $q \rightarrow 0$ фаза $\delta_{\lambda}^{-}$сходится к числу $-\alpha_{3} \pi / 2$, где $\alpha_{3}-$ знак коэффициента $a_{3}^{-}$или $r_{3}^{-}$, если $a_{3}^{-} \neq-6$ или $a_{3}^{-}=-6$. Из-за этой особенности фазы $\delta_{\lambda}^{-}$сечение $\sigma_{\lambda}^{-}$содержит квадрат функции $\sin \delta_{\lambda}^{\mathrm{c}}(-|\eta|)$ и растет, как $O\left(q^{-1}\right)$ :

$$
\sigma_{\lambda}^{\mathrm{int}}(-|\eta|) \sim 4 \frac{|R|}{q} \varepsilon_{\lambda} \sin ^{2} \delta_{\lambda}^{\mathrm{c}}(-|\eta|), \quad q \rightarrow 0
$$

\section{4. Асимптотики нормировочных множителей и волновых функций.} Рассмотрим поведение множителя $N^{ \pm}(q)$ при $q \rightarrow 0$. Для этого в определении (29) заменим функции $C_{\lambda}^{2}$ и $h$ их асимптотиками (17) и $(25)$, а функции $c^{ \pm}(v)$ и $s^{ \pm}(v)$ представим в виде $(71)$ с $m=1$. Затем воспользуемся свойствами предельных (при $v \rightarrow \infty)$ значений $c_{0}^{ \pm}$и $s_{0}^{ \pm}$компонент $c_{0}^{ \pm}(v)$ и $s_{0}^{ \pm}(v)$, а именно тем, что $c_{0}^{+} \neq 0$, а если $c_{0}^{-}=0$, то $s_{0}^{-} \neq 0$, и наоборот. Благодаря этим соотношениям в любом из случаев $1-3$, рассмотренных в пп. 5.2 и 5.3 , имеем

$$
N^{+}(q)=\frac{1}{\left|c_{0}^{ \pm}\right|}+O\left(q^{2}\right), \quad N^{-}(q)=\frac{1}{\sqrt{\left(c_{0}^{-}\right)^{2}+\left(\pi s_{0}^{-}\right)^{2}}}+O\left(q^{2}\right), \quad q \rightarrow 0 .
$$

Теперь исследуем радиальную волновую функцию $u_{\lambda}$, представленную формулами (32) через функции $f_{\lambda}^{ \pm}, \theta_{\lambda}^{ \pm}$и $c^{ \pm}, s^{ \pm}$. Как известно [4], в пределе $q \rightarrow 0$ при любом $v \geqslant 0$ разложения (33) функций $f_{\lambda}^{ \pm}$и $\theta_{\lambda}^{ \pm}$сходятся к компонентам $f_{\lambda 0}^{ \pm}$и $\theta_{\lambda 0}^{ \pm}$, которые согласно формулам (46) пропорциональны функциям Бесселя. В силу теоремы 7 в пределе $q \rightarrow 0$ разложения (34) функций $c^{ \pm}(v, q)$ и $s^{ \pm}(v, q)$ вырождаются в компоненты $c_{0}^{ \pm}(v)$ и $s_{0}^{ \pm}(v)$. Следовательно, в представлении (32) можно перейти к пределу $q \rightarrow 0$ и, используя приведенные выше асимптотики множителя $N^{ \pm}$, получить два предельных соотношения: в случае кулоновского отталкивания

$$
u_{\lambda 0}^{+}(v) \equiv \lim _{q \rightarrow 0} \frac{u_{\lambda}(\rho,+|\eta|}{q^{\lambda+1} C_{\lambda}(+|\eta|)}=\frac{\nu !}{2\left|c_{0}^{+}\right|}\left[c_{0}^{+}(v) I_{\nu}(v)+2 s_{0}^{+}(v) K_{\nu}(v)\right],
$$

а в случае кулоновского притяжения

$$
u_{\lambda 0}^{-}(v) \equiv \lim _{q \rightarrow 0} \frac{u_{\lambda}(\rho,-|\eta|)}{q^{\lambda+1} C_{\lambda}(-|\eta|)}=\frac{\nu !}{2 \sqrt{\left(c_{0}^{-}\right)^{2}+\left(\pi s_{0}^{-}\right)^{2}}}\left[c_{0}^{-}(v) J_{\nu}(v)-\pi s_{0}^{-}(v) Y_{\nu}(v)\right]
$$

Сформулируем два следствия этих предельных соотношений. Во-первых, произведение функций $q^{\lambda+1} C_{\lambda}( \pm|\eta|)$ и $u_{\lambda 0}^{ \pm}(v)$ является старшим слагаемым низкоэнергетической асимптотики волновой функции $u_{\lambda}(\rho, \pm|\eta|)$. Во-вторых, функция $u_{\lambda 0}^{ \pm}(v)$ является единственным решением исходной краевой задачи Шредингера (3)-(5) в случае нулевой полной энергии $E$.

\section{5. Условия существования длины рассеяния и эффективного радиу-}

са. Выясним, как должен убывать потенциал $V(v)$ в пределе $v \rightarrow \infty$, чтобы во всех случаях 1-3 длина рассеяния и эффективный радиус оказались конечными константами. Для этого используем соотношения (2), содержащие параметр $\beta$, и в каждом из случаев 1-3 найдем соответствующее число, которое этот параметр должен превышать. 
По определению короткодействующий потенциал $V$ удовлетворяет условиям (2) при любом сколь угодно большом значении параметра $\beta$. Поэтому для такого потенциала при любом сколь угодно большом номере $m$ леммы $1-3$ и теоремы 1-7 остаются в силе. Следовательно, разложения (71) существуют в пределе $m \rightarrow \infty$ и вырождаются в этом пределе в бесконечные ряды, а все компоненты $c_{n}^{ \pm}(v)$ и $s_{n}^{ \pm}(v)$, $n=0,1, \ldots$, этих рядов в точке $v=\infty$ принимают конечные значения $c_{n}^{ \pm}$и $s_{n}^{ \pm}$. Как показано в п. 5.2, длины рассеяния $a_{1}^{ \pm}, a_{2}^{ \pm}, a_{3}^{ \pm}$и эффективные радиусы $r_{1}^{ \pm}, r_{2}^{ \pm}$, $r_{3}^{ \pm}$выражаются через предельные значения $c_{n}^{ \pm}$и $s_{n}^{ \pm}, n=0,1,2$. Следовательно, в случае короткодействующего потенциала $V$ все такие длины рассеяния и эффективные радиусы существуют и являются конечными константами. Поэтому верны все асимптотические соотношения, выведенные в пп. 5.2-5.4.

Считая условия (1) выполненными, рассмотрим те же три случая, что и в п. 5.2.

СлучАй 1. Согласно формулам (80) длина рассеяния $a_{1}^{ \pm}=-s_{0}^{ \pm} / c_{0}^{ \pm}$, где $c_{0}^{ \pm}$и $s_{0}^{ \pm}-$ значения функций $c_{0}^{ \pm}(v)$ и $s_{0}^{ \pm}(v)$ в точке $v=\infty$. Как показано в теоремах 3 и 6 , такие значения существуют и являются конечными, если выполнены условия (8). Согласно последнему из них $V(v)=o\left(v^{-4}\right)$ при $v \rightarrow \infty$ для $\alpha=1$ или $\alpha=-1$. Теперь заметим, что коэффициент $-1 / a_{1}^{ \pm}$будет старшим слагаемым низкоэнергетической асимптотики (81) функции эффективного радиуса, если следующее слагаемое такой асимптотики будет сходиться к нулю, как $O\left(q^{2}\right)$. Для такой сходимости согласно теореме 7 должны существовать разбиения (71) с номером $m=1$. Для этого достаточным является условие $(2)$, в котором $2 \beta>3(m+1)$, a $m=1$. Следовательно, длина рассеяния $a_{1}^{ \pm}$существует, если в условии (2) параметр $\beta>3$. Приближения (84) и (85) фазы $\delta_{\lambda}^{ \pm}$и сечения $\sigma_{\lambda}^{ \pm}$содержат единственный параметр длину рассеяния $a_{1}^{ \pm}$. Поэтому эти приближения и все выведенные в п. 5.4 асимптотики справедливы при том же условии (2) с $\beta>3$. Если кулоновский потенциал $2 \eta / \rho$ является притягивающим $(\alpha=-1)$, то условию $(2)$ с $\beta>3$ удовлетворяет довольно медленно убывающий потенциал $V$, например потенциал с асимптотикой $V=O\left(v^{-2 p}\right)$ при $v \rightarrow \infty$, в которой $p>\beta>3$.

Теперь выявим условия, достаточные для существования эффективного радиуса $r_{1}^{ \pm}$. Согласно формулам (80) этот параметр представляется через значения $c_{n}^{ \pm}$ и $s_{n}^{ \pm}$функций $c_{n}^{ \pm}(v)$ и $s_{n}^{ \pm}(v)$ в точке $v=\infty$ (для $n \leqslant m=1$ ). Как показано в теоремах 3 и 6 , такие значения существуют и являются конечными при условиях (2), в которых $\beta>3(m+1)$, а $m=1$. Слагаемое $q^{2} r_{1}^{ \pm}$будет слагаемым низкоэнергетической асимптотики (81) функции эффективного радиуса, если следующее слагаемое такой асимптотики будет сходиться к нулю, как $O\left(q^{4}\right)$. Согласно теореме 7 для этого должны существовать разбиения (71) с номером $m=2$. Такие разбиения, а значит, и эффективный радиус $r_{1}^{ \pm}$, существуют при условиях $(2)$, в которых $2 \beta>3(m+1)$, a $m=2$. Эти условия являются достаточными и означают, что $\beta>9 / 2$.

СлучАи 2 и 3. Используя определения (82) и (83) функций эффективного радиуса и те же способы, что и в рассмотренном выше случае 1, нетрудно прийти к следующим выводам. Условия $(2)$, в которых $\beta>3(m+1)$, а $m=2$, являются достаточными для существования длин рассеяния $a_{2}^{ \pm}$и $a_{3}^{ \pm}$и приближений (86) фазы $\delta_{\lambda}^{ \pm}$и сечения $\sigma_{\lambda}^{ \pm}$. При условиях $(2)$, в которых $\beta>3(m+1)$, а $m=3$, заведомо существуют эффективные радиусы $r_{2}^{ \pm}$и $r_{3}^{ \pm}$, а фаза $\delta_{\lambda}^{-}$и сечение $\sigma_{\lambda}^{-}$имеют асимптотики (87). 


\section{6. ЗАКЛЮЧЕНИЕ}

В настоящей работе впервые дано расширение теории двумерного потенциального рассеяния на случай суперпозиции кулоновского и центрального короткодействующего потенциалов. В разделах 3 и 4 предложен и обоснован асимптотический в пределе низких энергий метод. Ключевой является рекуррентная по номеpy $n=0,1, \ldots$ и энергонезависимая цепочка линейных задач Коши $(35),(36)$ для компонент $c_{n}^{ \pm}$и $s_{n}^{ \pm}$. Доказано, что при любом значении квантового числа $\lambda$ эта цепочка однозначно разрешима в классе функций, непрерывных на полуоси $v \geqslant 0$. В разделе 5 предложенный метод применен для вывода и анализа низкоэнергетических приближений функции эффективного радиуса, радиальных волновых функций, парциальных фаз и сечений двумерного ядерно-кулоновского рассеяния. Такие приближения получены впервые и в качестве неизвестных функций содержат лишь компоненты $c_{n}^{ \pm}$и $s_{n}^{ \pm}$с $n \leqslant 2$, вычисление которых не может вызвать каких-либо принципиальных затруднений.

\section{Список литературы}

[1] D. Bollé, F. Gesztesy, "Scattering observables in arbitrary dimension $n \geqslant 2$ ", Phys. Rev. A, 30:3 (1984), 1279-1293.

[2] H. Friedrich, Scattering Theory, Lecture Notes in Physics, 872, Springer, Berlin, 2013.

[3] В. В. Бабиков, Метод фазовых функций в квантовой механике, Наука, М., 1976.

[4] В. В. Пупышев, “Двумерное кулоновское рассеяние квантовой частицы: строение радиальных волновых функций”, ТМФ, 186:1 (2016), 123-141.

[5] В.В. Пупышев, "Двумерное кулоновское рассеяние квантовой частицы: волновые функции и функции Грина”, ТМФ, 186:2 (2016), 252-271.

[6] В. В. Пупышев, "Двумерное кулоновское рассеяние квантовой частицы: низкоэнергетические асимптотики", ТМФ, 188:1 (2016), 49-75.

[7] В. В. Пупышев, "Метод амплитудных функций в теории двумерного рассеяния", ТМФ, 191:1 (2017), 34-62.

[8] Э. Камке, Справочник по обыкновенным дифференииалъным уравнениям, Наука, М., 1976.

[9] Г. Бейтмен, А. Эрдейи, Высшие трансцендентные функции, т. 2: Функиии Бесселя, функиии параболического иилиндра, ортогональные многочлены, Наука, М., 1974.

[10] Б. М. Будак, С. В. Фомин, Кратнъе интегралъ и ряды, Физматлит, М., 2002.

Поступила в редакцию 5.09.2016 